\title{
RIEMANNIAN F-MANIFOLDS, BI-FLAT F-MANIFOLDS, AND FLAT PENCILS OF METRICS
}

\author{
ALESSANDRO ARSIE, ALEXANDR BURYAK, PAOLO LORENZONI, AND PAOLO ROSSI
}

\begin{abstract}
In this paper we study relations between various natural structures on F-manifolds. In particular, given an arbitrary Riemannian F-manifold we present a construction of a canonical flat F-manifold associated to it. We also describe a construction of a canonical homogeneous Riemannian F-manifold associated to an arbitrary exact homogeneous flat pencil of metrics satisfying a certain non-degeneracy assumption. In the last part of the paper we construct Legendre transformations for Riemannian F-manifolds.
\end{abstract}

\section{CONTENTS}

Introduction

1. Riemannian F-manifolds and flat F-manifolds

1.1. From Riemannian F-manifolds to flat F-manifolds

1.2. From flat F-manifolds to Riemannian F-manifolds

1.3. An example: the Lobachevsky hyperbolic half-plane

1.4. A non-semisimple example in dimension 2

1.5. A non-semisimple example in dimension 3

2. Homogeneous Riemannian F-manifolds and bi-flat F-manifolds

2.1. Homogeneous flat F-manifolds and bi-flat F-manifolds

2.2. From homogeneous Riemannian F-manifolds to homogeneous flat F-manifolds

3. Riemannian F-manifolds with flat normal bundle

3.1. Riemannian F-manifolds with flat normal bundle and Hamiltonian operators

3.2. An example with a special Lauricella bi-flat F-manifold

3.3. An example with the Lobachevsky hyperbolic half-plane

4. Flat homogeneous Riemannian F-manifolds

4.1. The three-dimensional case

5. Exact homogeneous flat pencils of metrics

5.1. From flat pencils of metrics to homogeneous Riemannian F-manifolds

5.2. The semisimple case

5.3. Semisimple exact homogeneous flat pencils in dimension 3

6. Legendre transformations

6.1. The Legendre transformation for F-manifolds with compatible connection

6.2. The Legendre transformation for Riemannian F-manifolds

7. Appendix

References

\section{INTRODUCTION}

Since its beginnings, the field of integrable systems has interacted deeply with the study of differential geometric structures. A remarkable case of this interaction is provided by the notion of a semi-Hamiltonian system of hydrodynamic type introduced by Tsarev in [Tsa86, Tsa91]. These systems form a very wide class of integrable quasilinear system of PDEs of the form

$$
u_{t}^{i}=v^{i}(u) u_{x}^{i}, \quad i=1, \ldots, n .
$$

Date: April 20, 2021. 
The integrability is controlled by a set of $n(n-1)$ functions defined by the characteristic velocities of the system

$$
\Gamma_{i j}^{i}=\frac{\partial_{j} v^{i}}{v^{j}-v^{i}}, \quad i \neq j
$$

satisfying suitable conditions, called semi-Hamiltonian conditions [Tsa86, Tsa91] or richness conditions [Ser91]. As the notation suggests, the functions $\Gamma_{i j}^{i}$ can be identified with (a part of) the Christoffel symbols of a connection $\nabla$. A torsionless connection $\nabla$ can be reconstructed completely starting from $\Gamma_{i j}^{i}$ in essentially two different ways. The first one leads naturally to the notion of a Hamiltonian system of hydrodynamic type, while the second one leads to the notion of an F-manifold with compatible connection.

In the first case, starting from $\Gamma_{i j}^{i}$ one constructs a diagonal metric $g$ satisfying the conditions

$$
\partial_{j} \ln \sqrt{g_{i i}}=\Gamma_{i j}^{i}, \quad j \neq i,
$$

and all the remaining Christoffel symbols are uniquely defined through Levi-Civita's classical formula ( $\nabla$ is the Levi-Civita connection of the metric $g$ ). However, as it is easy to check, the general solution of (0.3) depends on $n$ arbitary functions of a single variable: if $g_{i i}$ is a solution then $\varphi_{i}\left(u^{i}\right) g_{i i}$ is still a solution.

Connections defined this way were introduced by Dubrovin and Novikov in [DN84]. We call them Hamiltonian connections since they are related to the Hamiltonian formalism. Indeed, when $\nabla$ is flat, the differential operator associated with the diagonal contravariant metric $g$

$$
P^{i j}:=g^{i i} \delta_{i}^{j} \partial_{x}-g^{i l} \Gamma_{l k}^{j} u_{x}^{k}
$$

defines a local Hamiltonian operator for the flow (0.1). In this case we call (0.1) a Hamiltonian system of hydrodynamic type. Conversely, given a flat non-degenerate pseudo-Riemannian metric $g_{i j}$, one can associate to it a local Poisson operator $P^{i j}$ as in (0.4). If the metrics defined by the solutions of system (0.3) are not flat, it is still possible to introduce a class of Hamiltonian operators of hydrodynamic type. The counterpart to the non-flatness of the metric is the non-locality of the associated Hamiltonian operator (see Fer91 for details). Integrability of the corresponding system is realized via the existence of sufficiently many functionals $F_{k}[u]$, $k=1,2, \ldots$, that Poisson commute with $H[u]$ (the Poisson bracket is induced via (0.4) and is called a Poisson bracket of hydrodynamic type). In some very important cases, the systems of hydrodynamic type (0.1) are not only Hamiltonian, but bi-Hamiltonian.

If a system of hydrodynamic type (10.1) is bi-Hamiltonian with respect to two Poisson brackets of hydrodynamic type induced by two local Hamiltonian operators, then the two corresponding flat contravariant metrics form a flat pencil of metrics (see [Dub98]).

Another way to reconstruct a torsionless affine connection $\nabla$ starting from $\Gamma_{i j}^{i}$ was developed in [LP11. Such connection and the product obtained identifying the Riemann invariants with the canonical coordinates lead naturally to introduce a class of F-manifolds, called F-manifolds with compatible connection [LPR11]. In the flat case these manifolds previously appeared in the literature under the name of Dubrovin manifolds Get04 and F-manifolds with compatible flat structure [Man05]. Following [LPR11] we will call them flat F-manifolds.

For a special class of integrable systems of hydrodynamic type the Hamiltonian connections and the natural connection coincide. They are called Egorov systems of hydrodynamic type [PT03] since the metrics satisfying system (0.3) are potential for a suitable choice of the Riemann invariants (depending on the chosen solution). Flat Egorov systems of hydrodynamic type were studied by Dubrovin in Dub90, and are called strongly integrable systems. In a sense, Dub90, can be seen as the dawn of what later would be the full-fledged theory of Dubrovin-Frobenius manifolds (known in the literature until recently simply as Frobenius manifolds). Indeed, in [Dub96] Egorov metrics appear as one of cornerstones of the vast landscape of Dubrovin-Frobenius manifolds. Dubrovin showed in Dub98, that starting from a flat pencil of metrics that satisfies three properties (exactness, homogeneity, and Egorov property) one can recover a Dubrovin-Frobenius manifold and coversely any Dubrovin-Frobenius manifold defines 
a flat pencil of metrics satisfying these properties. For instance, applying this construction to the Saito flat pencil of metrics associated with a Coxeter group [Sai93, SYS80] one gets the polynomial Dubrovin-Frobenius manifold structure on the orbit space of the group Dub98.

Many of the constructions appearing in the theory of Dubrovin-Frobenius manifolds have been generalized to the non-Egorov set-up, where Dubrovin-Frobenius manifolds are replaced by flat and bi-flat F-manifolds (in the conformal case). We refer to the papers [AL13, Lor14, KMS20, AL19, KM19] for relations with Painlevé trascendents, to the papers [AL17, KMS20, KMS18] for relations with reflection groups, to the papers ABLR20a, BB19] for relations with F-cohomological field theories, and to [AL18, ABLR20b for relations with integrable systems. In particular, the results of [ABLR20a combined with the results of [BR18] allow one to construct a generalization of double ramification hierarchy Bur15, BR16] for any semisimple flat F-manifold. The dispersionless limit of this hierarchy is the principal hierarchy associated with the given flat F-manifold. More general (but only first order and second order) dispersive deformations of the principal hierarchy have been considered in AL18.

In [AL13, Lor14 the construction of bi-flat F-manifolds was based on an (augmented) Darboux-Egorov system and the associated linear system of PDEs for the Lamé coefficients. The diagonal metric defined by the square of the Lamé coefficients was interpreted in ABLR20a as one of the data defining a semisimple Riemannian F-manifold (with Killing unit vector field).

This observation is the starting point of the present paper. The paper is divided into two parts. In the first part we investigate the relations between (homogeneous) Riemannian Fmanifolds and flat (Section 11) and bi-flat F-manifolds (Section 2) without semisimplicity assumption. This generalization is not straightforward and requires using explicitly the HertlingManin condition for F-manifolds HM99.

In the second part of the paper we study the system

$$
\begin{array}{rlrl}
\partial_{k} \beta_{i j} & =\beta_{i k} \beta_{k j}, & i \neq j \neq k \neq i, \\
\sum_{l=1}^{n} \partial_{l} \beta_{i j} & =0, & & i \neq j, \\
\sum_{l=1}^{n} u^{l} \partial_{l} \beta_{i j} & =-\beta_{i j}, & i \neq j,
\end{array}
$$

for functions $\beta_{i j}, i \neq j$, subject to the additional constraints

$$
\begin{array}{clrl}
\sum_{k \neq i, j}\left[\left(u^{j}-u^{k}\right)(\Delta \beta)_{i k} \beta_{j k}+\left(u^{k}-u^{i}\right)(\Delta \beta)_{j k} \beta_{i k}\right] & =(\Delta \beta)_{i j}, & i \neq j, \\
\left.\sum_{k \neq i, j}\left[u^{i}\left(u^{j}-u^{k}\right)(\Delta \beta)_{i k} \beta_{j k}-u^{j}\left(u^{i}-u^{k}\right)(\Delta \beta)_{j k} \beta_{i k}\right)\right] & =\frac{1}{2}\left(u^{i}+u^{j}\right)(\Delta \beta)_{i j}, & i \neq j,
\end{array}
$$

where $(\Delta \beta)_{i j}:=\beta_{i j}-\beta_{j i}$. In the Egorov case, i.e. when $(\Delta \beta)_{i j}=0$, the additonal constraints are automatically satisfied and the above system reduces to the system studied by Dubrovin in the theory of semisimple Dubrovin-Frobenius manifolds [Dub96]. In the non-Egorov case, the first constraint selects semisimple flat Riemannian F-manifolds. This case is of great importance in the theory of integrable systems because the flat metric defines a local Hamiltonian structure for the associated integrable hierarchies. The existence of a second compatible local Hamiltonian structure for these hierarchies requires that also the second constraint is satisfied. As an example, we focus on the three-dimensional case. It turns out that for any solution of the system above one can construct three different homogeneous Riemannian F-manifolds related by Legendre transformations. These transformations were first considered by Dubrovin [Dub96] in the case of Dubrovin-Frobenius manifolds and generalized later by Stedman and Strachan [SS17] to a more general framework. The definition given in this last paper can be promptly adapted to F-manifolds with compatible connection and in particular to flat F-manifolds, and we discuss it in Section 6 . 
Acknowledgements. The work of A. B. is supported by the Mathematical Center in Akademgorodok under agreement No. 075-15-2019-1675 with the Ministry of Science and Higher Education of the Russian Federation. P. L. is supported by funds of H2020-MSCA-RISE-2017 Project No. 778010 IPaDEGAN.

\section{Riemannian F-MANifolds AND Flat F-MANifolds}

Throughout the paper we will use the Einstein summation convention for repeated upper and lower indices, unless it is explicitly stated that such indices are fixed. Given a smooth manifold $M$ we denote by $C_{M}^{\infty}$ its sheaf of smooth functions and by $\mathcal{T}_{M}$ and $\Omega_{M}^{1}$ its tangent and cotangent sheaves. Moreover, given one of these sheaves $\mathcal{F}$, for brevity and at the cost of a slight abuse of notation, we will write $\sigma \in \mathcal{F}$ to mean $\sigma \in \mathcal{F}(U)$ for any (or some, depending on the context) open set $U \subset M$. In case several such sections appear in the same formula, the set $U$ is assumed to be the same for all of them.

F-manifolds have been introduced by Hertling and Manin in [HM99].

Definition 1.1. An F-manifold is a triple $(M, \circ, e)$, where $M$ is a manifold, $\circ$ is a commutative associative $C_{M}^{\infty}$-bilinear product o: $\mathcal{T}_{M} \times \mathcal{T}_{M} \rightarrow \mathcal{T}_{M}$ on the $C_{M}^{\infty}$-module $\mathcal{T}_{M}$ of (local) vector fields, satisfying the following identity:

$$
\begin{aligned}
& {[X \circ Y, W \circ Z]-[X \circ Y, Z] \circ W-[X \circ Y, W] \circ Z-X \circ[Y, Z \circ W]+X \circ[Y, Z] \circ W} \\
& +X \circ[Y, W] \circ Z-Y \circ[X, Z \circ W]+Y \circ[X, Z] \circ W+Y \circ[X, W] \circ Z=0,
\end{aligned}
$$

for all local vector fields $X, Y, W, Z$, where $[X, Y]$ is the Lie bracket, and $e$ is a distinguished vector field on $M$ such that $e \circ X=X$ for all local vector fields $X$. The requirement of having a unit vector field can be dropped, and in this case a pair $(M, \circ)$ will be called an $\mathrm{F}$-manifold without unit.

Remark 1.2. Condition (1.1) can be written in a more compact way as

$$
\mathcal{L}_{X \circ Y}(\circ)=X \circ \mathcal{L}_{Y}(\circ)+Y \circ \mathcal{L}_{X}(\circ), \quad X, Y \in \mathcal{T}_{M},
$$

where $\mathcal{L}_{X}$ denotes the Lie derivative.

If we express $\circ$ through a $(1,2)$ tensor $c$ with components $c_{j k}^{i}$ with respect to some local coordinate system on $M$, then condition (1.1) reads:

$$
c_{j l}^{q} \partial_{q} c_{s k}^{p}-c_{s k}^{q} \partial_{q} c_{j l}^{p}=c_{j q}^{p} \partial_{l} c_{s k}^{q}-c_{q k}^{p} \partial_{s} c_{j l}^{q}+c_{l q}^{p} \partial_{j} c_{s k}^{q}-c_{q s}^{p} \partial_{k} c_{j l}^{q} .
$$

Let us recall the following definition/notation.

Definition/Notation 1.3. Consider a manifold $M$ equipped with a commutative associative product $\circ$ in the tangent bundle.

1. The structure constants of the multiplication $\circ$ in some coordinate system will be denoted by $c_{j k}^{i}$.

2. A (pseudo-)Riemannian metric $g$ on $M$ is called invariant, or compatible with the product $\circ$, if

$$
g(X \circ Y, Z)=g(X, Y \circ Z), \quad X, Y, Z \in \mathcal{T}_{M} .
$$

In coordinates this condition reads $g_{i q} c_{l p}^{q}=g_{l q} c_{i p}^{q}$ or, equivalently, $g^{i q} c_{q p}^{l}=g^{l q} c_{q p}^{i}$.

3. A torsionless connection $\nabla$ on $M$ is said to be compatible with the product $\circ$, if $\left(\nabla_{X} c\right)(Y, Z)$ is symmetric in all of its entries. This is equivalent to the property that the Riemann tensor of the connection $\nabla^{(\lambda)}$ given by

$$
\nabla_{X}^{(\lambda)} Y:=\nabla_{X} Y+\lambda X \circ Y, \quad X, Y \in \mathcal{T}_{M},
$$

doesn't depend on $\lambda$. By a result of Hertling [Hert02, Theorem 2.14] this implies condition (1.1), and thus our manifold $M$ is an F-manifold in this case. 
4. If our manifold is equipped with a (pseudo-)Riemannian metric $g$ and a unit vector field for the product $\circ$, then the one-form $\theta$ on $M$ given by $\theta(X):=g(e, X), X \in \mathcal{T}_{M}$, is called the counit.

5. The derivative of a function $f$ along a vector field $X$ on $M$ will be denoted by $X(f)$.

Definition 1.4. A (pseudo-)Riemannian F-manifold is the datum of an F-manifold $(M, \circ, e)$ equipped with an invariant (pseudo-)Riemannian metric $g$ such that

$$
R(Y, Z)(X \circ W)+R(X, Y)(Z \circ W)+R(Z, X)(Y \circ W)=0, \quad X, Y, Z, W \in \mathcal{T}_{M},
$$

where $R$ is the Riemann tensor of $g$. If, additionally, the condition

$$
\mathcal{L}_{e} g=0
$$

is satisfied, then the manifold is called a (pseudo-)Riemannian F-manifold with Killing unit vector field.

In coordinates condition (1.4) reads

$$
R_{s k l}^{j} c_{m i}^{s}+R_{s m k}^{j} c_{l i}^{s}+R_{s l m}^{j} c_{k i}^{s}=0 .
$$

Remark 1.5. In the literature condition (1.4) is usually replaced by (see, e.g., [LPR11, Definition 17] and [DS11])

$$
Z \circ R(W, Y)(X)+W \circ R(Y, Z)(X)+Y \circ R(Z, W)(X)=0 .
$$

In two important cases the two conditions are equivalent:

- if $R$ is the Riemann tensor of a torsionless connection compatible with the product (see [LPR11, Remark 18]);

- if $R$ is the Riemann tensor of the Levi-Civita connection of an invariant metric. Indeed:

$$
\begin{aligned}
& g(R(W, Y)(X) \circ Z+R(Y, Z)(X) \circ W+R(Z, W)(X) \circ Y, \Lambda)= \\
= & g(R(W, Y)(X), Z \circ \Lambda)+g(R(Y, Z)(X), W \circ \Lambda)+g(R(Z, W)(X), Y \circ \Lambda)= \\
= & -g(R(W, Y)(Z \circ \Lambda)+R(Y, Z)(W \circ \Lambda)+R(Z, W)(Y \circ \Lambda), X) .
\end{aligned}
$$

Definition 1.6. A flat F-manifold (or an F-manifold with compatible flat structure) is a manifold $M$ equipped with a commutative associative product $\circ$ in the tangent bundle, a unit vector field $e$, and a torsionless connection $\nabla$ such that

(1) $\nabla e=0$,

(2) $\nabla$ is compatible with the product,

(3) $\nabla$ is flat.

As we already mentioned, the second condition implies that our manifold is indeed an Fmanifold.

Remark 1.7. Let us observe that in general a flat F-manifold is not a Riemannian F-manifold that happens to possess a flat metric (and hence a flat connection). The reason being that it's possible that none of the metrics $g$ compatible with $\nabla$ (i.e. satisfying the condition $\nabla g=0$ ) is compatible with the product in the sense of (1.3).

\subsection{From Riemannian F-manifolds to flat F-manifolds.}

Theorem 1.8. Let $(M, \circ, g, e)$ be a Riemannian F-manifold with Killing unit vector field.

1. There is a unique torsionless connection $\nabla$ on $M$ satisfying the condition

$$
\left(\nabla_{X} g\right)(Y, Z)=\frac{1}{2} d \theta(X \circ Y, Z)+\frac{1}{2} d \theta(X \circ Z, Y), \quad X, Y, Z \in \mathcal{T}_{M} .
$$

This connection is given by

$$
\nabla_{X} Y=\widetilde{\nabla}_{X} Y-\frac{1}{2}\left(\iota_{X \circ Y} d \theta\right)^{\sharp}, \quad X, Y \in \mathcal{T}_{M},
$$


where $\widetilde{\nabla}$ is the Levi-Civita connection associated to $g, \iota_{X}$ is the operator of contraction with the vector field $X$, and $\sharp: \Omega_{M}^{1} \rightarrow \mathcal{T}_{M}$ is the isomorphism between local one-forms and local vector fields induced by the cometric $g^{-1}$.

2. The tuple $(M, \circ, \nabla, e)$ defines a flat F-manifold.

Proof. For Part 1 the fact that the connection $\nabla$ given by (1.9) satisfies (1.8) is proved by a straightforward computation, while for the uniqueness of the connection see ABLR20a, proof of Theorem 1.13].

Let us prove Part 2. We have to check the three properties from Definition 1.6.

Let us prove that

$$
\nabla e=0 .
$$

Using (1.9) it is easy to see that

$$
g\left(\nabla_{X} e, Z\right)=g\left(\widetilde{\nabla}_{X} e, Z\right)-\frac{1}{2} d \theta(X, Z) .
$$

On the other hand,

$$
d \theta(X, Z)=X(g(e, Z))-Z(g(e, X))-g(e,[X, Z])
$$

and using

$$
\begin{aligned}
& 0=\left(\widetilde{\nabla}_{X} g\right)(e, Z)=X(g(e, Z))-g\left(\widetilde{\nabla}_{X} e, Z\right)-g\left(e, \widetilde{\nabla}_{X} Z\right), \\
& 0=\left(\widetilde{\nabla}_{Z} g\right)(e, X)=Z(g(e, X))-g\left(\widetilde{\nabla}_{Z} e, X\right)-g\left(e, \widetilde{\nabla}_{Z} X\right),
\end{aligned}
$$

and the fact that $[X, Z]=\widetilde{\nabla}_{X} Z-\widetilde{\nabla}_{Z} X$ in (1.11), it is easy to see that equation (1.10) becomes

$$
g\left(\nabla_{X} e, Z\right)=\frac{1}{2} g\left(\widetilde{\nabla}_{X} e, Z\right)+\frac{1}{2} g\left(\widetilde{\nabla}_{Z} e, X\right) .
$$

Let us show that the right-hand side of (1.12) vanishes identically. Indeed, from $\left(\mathcal{L}_{e} g\right)(X, Z)=0$ we have $e(g(X, Z))=g([e, X], Z)+g(X,[e, Z])$. On the other hand, from $\left(\widetilde{\nabla}_{e} g\right)(X, Z)=0$ we have also that $e(g(X, Z))=g\left(\widetilde{\nabla}_{e} X, Z\right)+g\left(X, \widetilde{\nabla}_{e} Z\right)$ and substituting this in $0=\left(\mathcal{L}_{e} g\right)(X, Z)$ we get that the right-hand side of (1.12) vanishes identically. Since $g$ is non-degenerate and $Z$ is arbitrary, we obtain $\nabla_{X} e=0$ for any local vector field $X$, as required.

Let us now prove that $\nabla$ is compatible with the product o, i.e. $\left(\nabla_{X} c\right)(Y, Z)=\left(\nabla_{Y} c\right)(X, Z)$, or, equivalently, $\nabla_{k} c_{l j}^{i}=\nabla_{l} c_{k j}^{i}$. We proceed as follows:

$$
\begin{aligned}
\nabla_{k} c_{l j}^{i}-\nabla_{l} c_{k j}^{i} & =\partial_{k} c_{l j}^{i}-\partial_{l} c_{k j}^{i}+\Gamma_{k m}^{i} c_{l j}^{m}-\Gamma_{k l}^{m} c_{m j}^{i}-\Gamma_{k j}^{m} c_{m l}^{i}-\Gamma_{l m}^{i} c_{k j}^{m}+\Gamma_{l k}^{m} c_{m j}^{i}+\Gamma_{l j}^{m} c_{m k}^{i}, \\
& =\partial_{k} c_{l j}^{i}-\partial_{l} c_{k j}^{i}+\Gamma_{k m}^{i} c_{l j}^{m}-\Gamma_{k j}^{m} c_{m l}^{i}-\Gamma_{l m}^{i} c_{k j}^{m}+\Gamma_{l j}^{m} c_{m k}^{i},
\end{aligned}
$$

where we have indicated with $\Gamma_{j k}^{i}$ the Christoffel symbols of the connection $\nabla$. We have

$$
\Gamma_{k l}^{i}=\widetilde{\Gamma}_{k l}^{i}-\frac{1}{2} g^{i f} c_{k l}^{q} d \theta_{q f}
$$

where $\theta_{i}=g_{i l} e^{l}, \widetilde{\Gamma}_{k l}^{i}$ are the Christoffel symbols of the Levi-Civita connection constructed from $g$, and $d \theta_{q f}=\partial_{q} \theta_{f}-\partial_{f} \theta_{q}$. In the expression (1.13) it is convenient to treat separately the contributions coming from the Levi-Civita connection of $g$, denote them by $A$, and those coming from the additional terms containing the counit, denote them by $B$ :

$$
\begin{aligned}
A= & \partial_{k} c_{l j}^{i}-\partial_{l} c_{k j}^{i}+\frac{1}{2} g^{i q}(\partial_{k} g_{q m}+\underbrace{\partial_{m} g_{q k}}_{* * *}-\partial_{q} g_{k m}) c_{l j}^{m}-\frac{1}{2} g^{m q}(\partial_{k} g_{q j}+\partial_{j} g_{q k}-\underbrace{\partial_{q} g_{k j}}_{* *}) c_{m l}^{i} \\
& -\frac{1}{2} g^{i q}(\partial_{l} g_{m q}+\underbrace{\partial_{m} g_{l q}}_{*}-\partial_{q} g_{l m}) c_{k j}^{m}+\frac{1}{2} g^{m q}(\partial_{l} g_{q j}+\partial_{j} g_{q l}-\underbrace{\partial_{q} g_{l j}}_{* * * *}) c_{m k}^{i}, \\
B= & -\frac{1}{2} g^{i s} c_{k m}^{q} d \theta_{q s} c_{l j}^{m}+\frac{1}{2} g^{m s} c_{k j}^{q} d \theta_{q s} c_{m l}^{i}+\frac{1}{2} g^{i s} c_{l m}^{q} d \theta_{q s} c_{k j}^{m}-\frac{1}{2} g^{m s} c_{l j}^{q} d \theta_{q s} c_{m k}^{i} .
\end{aligned}
$$


Using the associativity of the product, i.e. $c_{l m}^{q} c_{k j}^{m}=c_{k m}^{q} c_{l j}^{m}$, the first and the third terms in the last expression cancel out, and we remain with

$$
B=\frac{1}{2} g^{m s} c_{k j}^{q} c_{m l}^{i} \partial_{q} \theta_{s}-\frac{1}{2} g^{m s} c_{k j}^{q} c_{m l}^{i} \partial_{s} \theta_{q}-\frac{1}{2} g^{m s} c_{l j}^{q} c_{m k}^{i} \partial_{q} \theta_{s}+\frac{1}{2} g^{m s} c_{l j}^{q} c_{m k}^{i} \partial_{s} \theta_{q} .
$$

Since $g$ is compatible with the product $\circ$, we have

$$
B=\frac{1}{2} g^{m i} c_{k j}^{q} c_{m l}^{s} \partial_{q} \theta_{s}-\frac{1}{2} g^{m s} c_{k j}^{q} c_{m l}^{i} \partial_{s} \theta_{q}-\frac{1}{2} g^{m i} c_{l j}^{q} c_{m k}^{s} \partial_{q} \theta_{s}+\frac{1}{2} g^{m s} c_{l j}^{q} c_{m k}^{i} \partial_{s} \theta_{q} .
$$

Now we integrate by parts obtaining:

$$
\begin{aligned}
B= & \frac{1}{2} g^{m i} c_{k j}^{q} \partial_{q}\left(c_{m l}^{s} \theta_{s}\right)-\frac{1}{2} g^{m i} c_{k j}^{q} \theta_{s} \partial_{q} c_{m l}^{s}-\frac{1}{2} g^{m s} c_{m l}^{i} \partial_{s}\left(c_{k j}^{q} \theta_{q}\right)+\frac{1}{2} g^{m s} c_{m l}^{i} \theta_{q} \partial_{s} c_{k j}^{q} \\
& -\frac{1}{2} g^{m i} c_{l j}^{q} \partial_{q}\left(c_{m k}^{s} \theta_{s}\right)+\frac{1}{2} g^{m i} c_{l j}^{q} \theta_{s} \partial_{q} c_{m k}^{s}+\frac{1}{2} g^{m s} c_{m k}^{i} \partial_{s}\left(c_{l j}^{q} \theta_{q}\right)-\frac{1}{2} g^{m s} c_{m k}^{i} \theta_{q} \partial_{s} c_{l j}^{q} .
\end{aligned}
$$

Now observe that $\theta_{p} c_{s l}^{p}=g_{p r} e^{r} c_{s l}^{p}$, and using the compatibility of $g$ with $\circ$ this is equal to $g_{p s} e^{r} c_{r l}^{p}=g_{p s} \delta_{l}^{p}=g_{s l}$, since $e$ is the unit of $\circ$, and analogously for other indices. Therefore, $B$ simplifies to

$$
\begin{aligned}
B= & \underbrace{\frac{1}{2} g^{m i} \partial_{q} g_{m l} c_{k j}^{q}}_{*}-\underbrace{\frac{1}{2} g^{m s} \partial_{s} g_{k j} c_{m l}^{i}}_{* *}-\underbrace{\frac{1}{2} g^{m i} \partial_{q} g_{m k} c_{l j}^{q}}_{* * *}+\underbrace{\frac{1}{2} g^{m s} \partial_{s} g_{l j} c_{m k}^{i}}_{* * * *} \\
& +\frac{1}{2} g^{m i} \theta_{s}\left[c_{l j}^{q} \partial_{q} c_{m k}^{s}-c_{k j}^{q} \partial_{q} c_{m l}^{s}\right]+\frac{1}{2} g^{m s} \theta_{q}\left[c_{m l}^{i} \partial_{s} c_{k j}^{q}-c_{m k}^{i} \partial_{s} c_{l j}^{q}\right] .
\end{aligned}
$$

Note that the first four terms of $B$ cancel out with four terms of $A$, and we are left with $A+B=C+D$, where

$$
\begin{aligned}
C= & \partial_{k} c_{l j}^{i}-\partial_{l} c_{k j}^{i}+\frac{1}{2} g^{i m}\left(\partial_{k} g_{m q}-\partial_{m} g_{k q}\right) c_{l j}^{q}-\frac{1}{2} g^{m s}\left(\partial_{k} g_{s j}+\partial_{j} g_{s k}\right) c_{m l}^{i}-\frac{1}{2} g^{i m}\left(\partial_{l} g_{q m}-\partial_{m} g_{l q}\right) c_{k j}^{q} \\
& +\frac{1}{2} g^{m s}\left(\partial_{l} g_{s j}+\partial_{j} g_{s l}\right) c_{m k}^{i}, \\
D= & \frac{1}{2} g^{m i} \theta_{s}\left[c_{l j}^{q} \partial_{q} c_{m k}^{s}-c_{k j}^{q} \partial_{q} c_{m l}^{s}\right]+\frac{1}{2} g^{m s} \theta_{q}\left[c_{m l}^{i} \partial_{s} c_{k j}^{q}-c_{m k}^{i} \partial_{s} c_{l j}^{q}\right] .
\end{aligned}
$$

To conclude, we rewrite $D$ using the Hertling-Manin condition (1.2). Renaming summed indices and using the compatibility of $g$ with $\circ$ in the second term (i.e. $g^{m s} c_{m l}^{i}=g^{m i} c_{m l}^{s}$ and analogously for the other monomial in the second term), we obtain:

$$
\begin{aligned}
D & =\frac{1}{2} g^{i s} \theta_{p}\left[c_{l j}^{q} \partial_{q} c_{s k}^{p}-c_{k j}^{q} \partial_{q} c_{s l}^{p}\right]+\frac{1}{2} g^{i s} \theta_{p}\left[c_{s l}^{q} \partial_{q} c_{k j}^{p}-c_{s k}^{q} \partial_{q} c_{l j}^{p}\right]= \\
& =\frac{1}{2} g^{i s} \theta_{p}\left[c_{l j}^{q} \partial_{q} c_{s k}^{p}-c_{s k}^{q} \partial_{q} c_{l j}^{p}\right]-\frac{1}{2} g^{i s} \theta_{p}\left[c_{k j}^{q} \partial_{q} c_{s l}^{p}-c_{s l}^{q} \partial_{q} c_{k j}^{p}\right],
\end{aligned}
$$

where in the last line we have rearranged the terms in order to apply (1.2) to the expressions inside square brackets. Applying (1.2) and using the fact that $\theta_{p} c_{j q}^{p}=g_{j q}$ (and analogously for other indices), we obtain:

$$
\begin{aligned}
D= & \frac{1}{2} g^{i s}\left[g_{j q} \partial_{l} c_{s k}^{q}-g_{q k} \partial_{s} c_{j l}^{q}+g_{l q} \partial_{j} c_{s k}^{q}-g_{q s} \partial_{k} c_{j l}^{q}\right]-\frac{1}{2} g^{i s}\left[g_{j q} \partial_{k} c_{s l}^{q}-g_{q l} \partial_{s} c_{j k}^{q}+g_{k q} \partial_{j} c_{s l}^{q}-g_{q s} \partial_{l} c_{j k}^{q}\right] \\
= & \frac{1}{2} g^{i s}\left[\partial_{l}\left(g_{j q} c_{s k}^{q}\right)-c_{s k}^{q} \partial_{l} g_{j q}-\partial_{s}\left(g_{q k} c_{j l}^{q}\right)+c_{j l}^{q} \partial_{s} g_{q k}+\partial_{j}\left(g_{t q} c_{s k}^{q}\right)-c_{s k}^{q} \partial_{j} g_{l q}\right]-\frac{1}{2} \partial_{k} c_{j l}^{i} \\
& -\frac{1}{2} g^{i s}\left[\partial_{k}\left(g_{j q} c_{s l}^{q}\right)-c_{s l}^{q} \partial_{k}\left(g_{j q}\right)-\partial_{s}\left(g_{q l} c_{j k}^{q}\right)+c_{j k}^{q} \partial_{s} g_{q l}+\partial_{j}\left(g k q c_{s l}^{q}\right)-c_{s l}^{q} \partial_{j} g_{k q}\right]+\frac{1}{2} \partial_{l} c_{j k}^{i},
\end{aligned}
$$

where we have performed integration by parts and used the invariance of $g$ with respect to $\circ$. Now in the expression for $D$ we write $\partial_{l}\left(g_{j q} c_{s k}^{q}\right)=\partial_{l}\left(g_{s q} c_{j k}^{q}\right)$ and expand the expression using 
the Leibnitz rule and do the same with $\partial_{k}\left(g_{j q} c_{s l}^{q}\right)$ :

$$
\begin{aligned}
D= & \frac{1}{2} g^{i s}\left[c_{j k}^{q} \partial_{l} g_{s q}-c_{s k}^{q} \partial_{l} g_{j q}+c_{j l}^{q} \partial_{s} g_{q k}-c_{s k}^{q} \partial_{j} g_{l q}\right]+\frac{1}{2} \partial_{l} c_{j k}^{i}-\frac{1}{2} \partial_{k} c_{j l}^{i} \\
& -\frac{1}{2} g^{i s}\left[c_{j l}^{q} \partial_{k} g_{s q}-c_{s l}^{q} \partial_{k}\left(g_{j q}\right)+c_{j k}^{q} \partial_{s} g_{q l}-c_{s l}^{q} \partial_{j} g_{k q}\right]-\frac{1}{2} \partial_{k} c_{j l}^{i}+\frac{1}{2} \partial_{l} c_{j k}^{i} .
\end{aligned}
$$

To compare more effectively $C$ and $D$, we rewrite $C$ as

$$
\begin{aligned}
C= & \partial_{k} c_{l j}^{i}-\partial_{l} c_{k j}^{i}+\frac{1}{2} g^{i s}\left(\partial_{k} g_{s q}-\partial_{s} g_{k q}\right) c_{l j}^{q}-\frac{1}{2} g^{i s}\left(\partial_{k} g_{q j}+\partial_{j} g_{q k}\right) c_{s l}^{q} \\
& -\frac{1}{2} g^{i s}\left(\partial_{l} g_{q s}-\partial_{s} g_{l q}\right) c_{k j}^{q}+\frac{1}{2} g^{i s}\left(\partial_{l} g_{q j}+\partial_{j} g_{q l}\right) c_{s k}^{q},
\end{aligned}
$$

where we have renamed summed indexes and used the invariance of $g$ with respect to $\circ$. It is now immediate to see that $C+D=0$, thus proving that $\nabla$ is compatible with the product $\circ$.

Before proving that $\nabla$ is flat, let us prove some preliminary lemmas.

Lemma 1.9. Let $M$ be a manifold equipped with a torsionless connection $\nabla$ and a vector field $X$ such that $\nabla X=0$. Then $\mathcal{L}_{X} T=\nabla_{X} T$ for any tensor field $T$ on $M$.

Proof. Note that for any vector field $Y$ we have $\nabla_{X} Y=\mathcal{L}_{X} Y+\nabla_{Y} X=\mathcal{L}_{X} Y$. Therefore, if $T$ is a $(k, l)$ tensor, then

$$
\begin{aligned}
\left(\mathcal{L}_{X} T\right)\left(Y_{1}, \ldots, Y_{l}\right) & =\mathcal{L}_{X}\left(T\left(Y_{1}, \ldots, Y_{l}\right)\right)-\sum_{i=1}^{l} T\left(Y_{1}, \ldots, \mathcal{L}_{X} Y_{i}, \ldots, Y_{l}\right)= \\
& =\nabla_{X}\left(T\left(Y_{1}, \ldots, Y_{l}\right)\right)-\sum_{i=1}^{l} T\left(Y_{1}, \ldots, \nabla_{X} Y_{i}, \ldots, Y_{l}\right)=\left(\nabla_{X} T\right)\left(Y_{1}, \ldots, Y_{l}\right),
\end{aligned}
$$

as required.

Lemma 1.10. Consider a manifold $M$ equipped with a commutative associative product $\circ$ and a unit vector field e.

1. If $M$ is equipped with a (pseudo-)Riemannian metric g such that $\mathcal{L}_{e} g=0$, then $\mathcal{L}_{e} \theta=0$.

2. If $M$ is equipped with a torsionless connection $\nabla$ compatible with the product $\circ$ and such that $\nabla e=0$, then $\mathcal{L}_{e} c=0$.

Proof. For Part 1 we compute

$$
\left(\mathcal{L}_{e} \theta\right)(Y)=e(\theta(Y))-\theta\left(\mathcal{L}_{e} Y\right)=\left(\mathcal{L}_{e} g\right)(e, Y)+g\left(\mathcal{L}_{e} e, Y\right)+g\left(e, \mathcal{E}_{e} Y\right)-\underline{\theta}\left(\mathcal{L}_{e} Y\right)=0,
$$

and for Part 2:

$\left(\mathcal{L}_{e} c\right)(X, Y) \stackrel{\text { Lemma } 1.9}{=}\left(\nabla_{e} c\right)(X, Y)=\left(\nabla_{X} c\right)(e, Y)=\nabla_{X}(c(e, Y))-c\left(\nabla_{X} e, Y\right)-c\left(e, \nabla_{X} Y\right)=0$.

Lemma 1.11. Consider a manifold $M$ equipped with a commutative associative product $\circ$, a unit vector field $e$, and a torsionless connection $\nabla$. Then $\nabla$ is flat if and only if the curvature operator $R$ of $\nabla$ satisfies condition (1.4) together with the condition

$$
R(e, X)=0, \quad X \in \mathcal{T}_{M} .
$$

Proof. Substituting $Y=e$ in (1.4) and using (1.14) and the fact that $e$ is the unit for $\circ$, one obtains immediately that $R(Z, X)(W)=0$ for all local vector fields $X, Z, W$. The converse statement is obvious.

Proposition 1.12. Consider a manifold $M$ equipped with a commutative associative product $\circ$ and two connections $\nabla, \widetilde{\nabla}$ such that $\nabla$ is compatible with the product and

$$
\nabla_{X} Y=\widetilde{\nabla}_{X} Y+W(X \circ Y), \quad X, Y \in \mathcal{T}_{M},
$$


for some $(1,1)$ tensor field $W$. Then

$$
\begin{aligned}
& R(Y, Z)(X \circ W)+R(X, Y)(Z \circ W)+R(Z, X)(Y \circ W)= \\
= & \widetilde{R}(Y, Z)(X \circ W)+\widetilde{R}(X, Y)(Z \circ W)+\widetilde{R}(Z, X)(Y \circ W), \quad X, Y, Z, W \in \mathcal{T}_{M},
\end{aligned}
$$

where $R, \widetilde{R}$ and the Riemann tensors for the connections $\nabla, \widetilde{\nabla}$, respectively.

Proof. Denote by $\Gamma_{j k}^{j}$ the Christoffel symbols of the connection $\nabla$ and by $a_{j k}^{h}$ the Christoffel symbols of the connection $\widetilde{\nabla}$. We have

$$
\Gamma_{j k}^{h}:=a_{j k}^{h}+b_{j k}^{h},
$$

where $b_{j k}^{j}=c_{j k}^{s} W_{s}^{h}$ and, therefore,

$$
\begin{aligned}
R_{i k j}^{h} & =\widetilde{R}_{i k j}^{h}+\partial_{k} b_{i j}^{h}-\partial_{j} b_{i k}^{h}+a_{i j}^{s} b_{k s}^{h}-a_{i k}^{s} b_{j s}^{h}+b_{i j}^{s} a_{k s}^{h}-b_{i k}^{s} a_{j s}^{h}+b_{i j}^{s} b_{k s}^{h}-b_{i k}^{s} b_{j s}^{h}= \\
& =\widetilde{R}_{i k j}^{h}+\nabla_{k} b_{i j}^{h}-\nabla_{j} b_{i k}^{h}+b_{s j}^{h} b_{i k}^{s}-b_{s k}^{h} b_{i j}^{s}=: \widetilde{R}_{i k j}^{h}+T_{i k j}^{h} .
\end{aligned}
$$

Using the condition $\nabla_{k} c_{j l}^{i}=\nabla_{j} c_{k l}^{i}$ it is immediate to prove that

$$
T_{i k j}^{h}=\left(c_{i j}^{m} \nabla_{k} W_{m}^{h}-c_{i k}^{m} \nabla_{j} W_{m}^{h}\right)+W_{l}^{s} W_{m}^{h}\left(c_{i k}^{l} c_{s j}^{m}-c_{i j}^{l} c_{s k}^{m}\right)
$$

or, equivalently,

$$
\begin{aligned}
R(X, Y)(Z)=\widetilde{R}(X, Y)(Z) & {\left[\left(\nabla_{X} W\right)(Z \circ Y)-\left(\nabla_{Y} W\right)(Z \circ X)\right] } \\
+ & {[W(W(Z \circ X) \circ Y)-W(W(Z \circ Y) \circ X)], \quad X, Y, Z \in \mathcal{T}_{M}, }
\end{aligned}
$$

which implies (1.15) via a simple straightforward computation.

We are now ready to prove that $\nabla$ is flat. Denote by $\widehat{R}$ the Riemann tensor of $\nabla$. By Lemma 1.11 it is sufficient to check that $\widehat{R}$ satisfies condition (1.4) together with condition (1.14). For the $(1,1)$ tensor field $W$ on $M$ defined by $W(X):=-\frac{1}{2}\left(\iota_{X} d \theta\right)^{\sharp}$ we have $\nabla_{X} Y=\widetilde{\nabla}_{X} Y+W(X \circ Y)$ and, therefore, by Proposition 1.12 condition (1.4) is true for the tensor $\widehat{R}$, since this condition is true for the tensor $R$.

It remains to check that $\widehat{R}(e, X)=0$ or, equivalently,

$$
\widehat{R}_{i k j}^{h} e^{j}=0 \text {. }
$$

We have

$$
\widehat{R}_{i k j}^{h} e^{j}=e^{j} \partial_{k} \Gamma_{i j}^{h}-e\left(\Gamma_{i k}^{h}\right)+\Gamma_{i j}^{s} e^{j} \Gamma_{k s}^{h}-\Gamma_{i k}^{s} \Gamma_{j s}^{h} e^{j},
$$

Using the condition $\nabla e=0$ we can reduce the above expression to

$$
\widehat{R}_{i k j}^{h} e^{j}=-e\left(\Gamma_{i k}^{h}\right)-\partial_{k} \partial_{i} e^{h}-\Gamma_{i s}^{h} \partial_{k} e^{s}-\Gamma_{k s}^{h} \partial_{i} e^{s}+\Gamma_{i k}^{s} \partial_{s} e^{h} .
$$

Let us express

$$
\Gamma_{i k}^{h}=\widetilde{\Gamma}_{i k}^{h}+b_{i k}^{h}, \quad \text { where } \quad b_{i k}^{h}=-\frac{1}{2} g^{h s} c_{i k}^{l} d \theta_{l s} .
$$

Using that $\mathcal{L}_{e} g=0$ it is not difficult to prove that

$$
e\left(\widetilde{\Gamma}_{i k}^{h}\right)=\widetilde{\Gamma}_{i k}^{s} \partial_{s} e^{h}-\widetilde{\Gamma}_{i m}^{h} \partial_{k} e^{m}-\widetilde{\Gamma}_{k m}^{h} \partial_{i} e^{m}-\partial_{i} \partial_{k} e^{h} .
$$

Similarly using $\mathcal{L}_{e} g=0$, and the conditions $\mathcal{L}_{e} \theta=0$ and $\mathcal{L}_{e} c=0$, which hold because of Lemma 1.10, one can prove that

$$
e\left(b_{i k}^{h}\right)=b_{i k}^{s} \partial_{s} e^{h}-b_{i m}^{h} \partial_{k} e^{m}-b_{k m}^{h} \partial_{i} e^{m} .
$$

Combining the above relations we get

$$
e\left(\Gamma_{i k}^{h}\right)=\Gamma_{i k}^{s} \partial_{s} e^{h}-\Gamma_{i m}^{h} \partial_{k} e^{m}-\Gamma_{k m}^{h} \partial_{i} e^{m}-\partial_{i} \partial_{k} e^{h},
$$

and substituting this on the right-hand side of (1.16) we get $\widehat{R}_{i k j}^{h} e^{j}=0$, as required. 
Let us remark that in Theorem 1.8 no assumption is made about the semisimplicity of the product $\circ$ or even its regularity in the sense of David-Hertling (see [DH17]). The above theorem was proved in [ABLR20a, Theorem 1.13] assuming that the product is semisimple.

1.2. From flat F-manifolds to Riemannian F-manifolds. Now we try to reconstruct a Riemannian F-manifold with Killing unit vector field starting from a flat F-manifold.

Theorem 1.13. Let $(M, \circ, \nabla, e)$ be a flat $F$-manifold and let $g$ be an invariant metric satisfying condition (1.8). Then the tuple $(M, \circ, g, e)$ defines a Riemannian F-manifold with Killing unit vector field.

Proof. The proof is based on the following lemma.

Lemma 1.14. Let $(M, \circ, \nabla, e)$ be a flat $F$-manifold and $g$ be any metric satisfying condition (1.8).

1. We have $\mathcal{L}_{e} g=0$.

2. Let $\widetilde{\nabla}$ be the connection defined by

$$
\widetilde{\nabla}_{X} Y:=\nabla_{X} Y+\frac{1}{2}\left(\iota_{X \circ Y} d \theta\right)^{\sharp} .
$$

Then $\widetilde{\nabla} g=0$.

3. The Riemann tensor of $g$ satisfies condition (1.4).

Proof. Part 1:

$$
\left(\mathcal{L}_{e} g\right)(Y, Z) \stackrel{\text { Lemma } 1.9}{=}\left(\nabla_{e} g\right)(Y, Z) \stackrel{(1.8)}{=} \frac{1}{2} d \theta(Y, Z)+\frac{1}{2} d \theta(Z, Y)=0
$$

by skewsymmetry of $d \theta$.

Part 2:

$$
\begin{aligned}
\left(\widetilde{\nabla}_{X} g\right)(Y, Z) & =X(g(Y, Z))-g\left(\widetilde{\nabla}_{X} Y, Z\right)-g\left(Y, \widetilde{\nabla}_{X} Z\right)= \\
& =\left(\nabla_{X} g\right)(Y, Z)+g\left(\nabla_{X} Y, Z\right)+g\left(Y, \nabla_{X} Z\right)-g\left(\widetilde{\nabla}_{X} Y, Z\right)-g\left(Y, \widetilde{\nabla}_{X} Z\right)= \\
& =\left(\nabla_{X} g\right)(Y, Z)-\frac{1}{2} d \theta(X \circ Y, Z)-\frac{1}{2} d \theta(X \circ Z, Y) \stackrel{(1.8)}{=} \\
& =0 .
\end{aligned}
$$

Part 3 immediately follows from Part 2 and Proposition 1.12 , since $\nabla$ is flat.

The theorem obviously follows from the lemma.

Remark 1.15. In the case of a semisimple flat F-manifold, in canonical coordinates, an invariant metric is diagonal, $g_{i j}=\delta_{j}^{i} g_{i i}$, and system (1.8) reads

$$
\delta_{j}^{i} \partial_{k} g_{i i}-\Gamma_{k i}^{j} g_{j j}-\Gamma_{k j}^{i} g_{i i}=\frac{1}{2}\left(\delta_{k}^{i}-\delta_{k}^{j}\right)\left(\partial_{i} g_{j j}-\partial_{j} g_{i i}\right), \quad 1 \leq i, j, k \leq \operatorname{dim} M .
$$

This is a complete compatible system whose general solution depends on $n=\operatorname{dim} M$ arbitrary constants (see [ABLR20a, Proposition 1.8] and the paragraph before).

1.3. An example: the Lobachevsky hyperbolic half-plane. We conclude this section with an example of Riemannian F-manifold with Killing unit vector field. Consider the following (rotated) version of the Lobachevsky hyperbolic half-plane: $\mathcal{H}:=\left\{(x, y) \in \mathbb{R}^{2} \mid x>y\right\}$ with the metric $g:=\frac{2}{(x-y)^{2}}\left(d x^{2}+d y^{2}\right)$. We declare $(x, y)$ to be canonical coordinates for a semisimple product $\circ$, so that in these coordinates $c_{j k}^{i}=\delta_{j}^{i} \delta_{k}^{i}$. The unit vector field is $e=\partial_{x}+\partial_{y}$. It is immediate to check that $\mathcal{L}_{e} g=0$ and that $g$ is invariant. Finally, condition (1.6) in this case reads:

$$
R_{s k l}^{j} \delta_{m}^{s} \delta_{i}^{s}+R_{s m k}^{j} \delta_{l}^{s} \delta_{i}^{s}+R_{s l m}^{j} \delta_{k}^{s} \delta_{i}^{s}=0, \quad 1 \leq k, l, m, i, j \leq 2,
$$

which is clearly satisfied if $k, l, m \neq i$. Otherwise, since condition (1.6) is symmetric with respect to cyclic permutations of $k, l, m$, we can assume that $m=i$. If also $l, k \neq i$, then 
automatically $l=k$ (because $\operatorname{dim} \mathcal{H}=2$ ) and the condition reduces to $R_{m k k}^{j}=0$, which is true by skewsymmetry. If exactly two indices from $k, l, m$ are equal to $i$, then we can assume that $i=m=l \neq k$, and the condition reduces to $R_{i k i}^{j}+R_{i i k}^{j}=0$, which is also satisfied by skewsymmetry. If $i=m=k=l$, then all the terms in the constraint are equal to $R_{i i i}^{j}=0$, again by skewsymmetry. Therefore, $(\mathcal{H}, \circ, g, e)$ is a semisimple Riemannian F-manifold with Killing unit vector field.

It is easy to compute that flat coordinates of the associated flat F-manifold are given by $t^{1}=\frac{4}{x-y}, t^{2}=\frac{x+y}{2}$, and a corresponding vector potential is $\left(F^{1}, F^{2}\right)=\left(t^{1} t^{2}, \frac{\left(t^{2}\right)^{2}}{2}+\frac{2}{3}\left(t^{1}\right)^{-2}\right)$. The unit vector field is $\frac{\partial}{\partial t^{2}}$.

In Section 3.3 we will see that the Lobachevsky hyperbolic half-plane is an example of a Riemannian F-manifold with flat normal bundle.

1.4. A non-semisimple example in dimension 2. Let us consider the flat structure defined in David-Hertling canonical coordinates $(x, y)$ (see [DH17]) by the data

$$
c_{i j}^{k}=\delta_{i+j-1}^{k}, \quad e=\partial_{x}, \quad E=x \partial_{x}+y \partial_{y},
$$

and by the Christoffel symbols (only non vanishing symbols are listed)

$$
\Gamma_{22}^{1}=\frac{b}{y}, \quad \Gamma_{22}^{2}=\frac{a}{y},
$$

where $a$ and $b$ are constants (we list in the Appendix the vector potentials associated with this family). It is easy to check that the associated metrics exist only if $b=0$ and are given by

$$
g=\left[\begin{array}{cc}
f(y) & c y^{a} \\
c y^{a} & 0
\end{array}\right]
$$

where $c$ is an abitrary constant and $F(y)$ is an arbitrary function.

1.5. A non-semisimple example in dimension 3. Let us consider the flat structure defined in David-Hertling canonical coordinates $(x, y, z)$ by the data

$$
c_{i j}^{k}=\delta_{i+j-1}^{k}, \quad e=\partial_{x}, \quad E=x \partial_{x}+y \partial_{y}+z \partial_{z},
$$

and by the Christoffel symbols (only non-vanishing symbols are listed)

$$
\Gamma_{23}^{3}=\Gamma_{32}^{3}=\frac{a}{y}, \quad \Gamma_{22}^{3}=\frac{(a b+2 b) y-2 a z}{(a+2)} \frac{1}{y^{2}}, \quad \Gamma_{22}^{2}=\frac{a(a+1)}{(a+2)} \frac{1}{y},
$$

where $a \neq 2$ and $b$ are constants (see AL19, Theorem 5.10]). The associated invariant metrics satisfying (1.8) (obtained using the computational software Maple) have the form

$$
g=\left[\begin{array}{ccc}
g_{11} & g_{12} & g_{13} \\
g_{21} & g_{22} & 0 \\
g_{31} & 0 & 0
\end{array}\right],
$$

where

$$
\begin{aligned}
& g_{11}=2 F^{\prime}(y) y z+G(y) y+\frac{2}{9}\left(\frac{a^{2}\left(a^{2}+3 a+3\right)}{(a+2)^{2}}\right) c y^{\frac{4}{3} \frac{a^{2}-3}{a+2}} z^{2}-2\left(b c y^{\frac{1}{3} \frac{4 a^{2}+3 a-6}{a+2}}+\frac{a^{2}-2}{a+2} F(y)\right) z, \\
& g_{12}=g_{21}=\frac{2}{3}\left(\frac{a(a+3)}{a+2}\right) c y^{\frac{1}{3} \frac{4 a^{2}+3 a-6}{a+2} z+y F(y),} \\
& g_{13}=g_{22}=g_{31}=c y^{\frac{2}{3} \frac{(2 a+3) a}{a+2}} .
\end{aligned}
$$

In the above formulas $c$ is an arbitrary constant while $F(y)$ and $G(y)$ are arbitrary functions. 


\section{Homogeneous Riemannian F-Manifolds and Bi-Flat F-manifolds}

In this section we consider flat F-manifolds equipped with a second distinguished vector field, called the Euler vector field. In the literature one can find three different and (a posteriori) equivalent ways to do this:

(1) Dropping the axioms involving explicitly the metric apart from those involving only the Levi-Civita connection in the definition of a (conformal) Dubrovin-Frobenius manifold. This is the most straightforward way and leads to the definition of a Dubrovin-Frobenius manifold without metric [AL17] or flat F-manifold with (linear) Euler vector field [DH19] or homogeneous flat F-manifold [ABLR20a, (see also ABLR20b, Remark 2.2]).

(2) In terms of a flat meromorphic connection on the bundle $\pi^{*} T M$ on $\mathbb{P} \times M$ called Saito structure without metric (see [Sab98] for details).

(3) In terms of a pair of compatible flat structures. This generalizes the notion of compatible flat metrics (flat pencil of metrics) and leads to the notion of a bi-flat F-manifold.

\subsection{Homogeneous flat F-manifolds and bi-flat F-manifolds.}

Definition 2.1. ABLR20a] $A$ homogeneous flat F-manifold (also called a manifold with Saito structure in [KMS18]) is a flat $F$-manifold $(M, \circ, \nabla, e)$ equipped with a vector field $E$, called the Euler vector field, satisfying $\mathcal{L}_{E^{\circ}}=\circ$ and $\nabla \nabla E=0$.

Definition 2.2. AL13] A bi-flat F-manifold is a manifold $M$ equipped with two different flat $F$-manifold structures $(\circ, \nabla, e)$ and $\left(*, \nabla^{*}, E\right)$ related by the following conditions:

(1) $\mathcal{L}_{E}$ O $=$;

(2) $X * Y=(E \circ)^{-1} X \circ Y$ for all local vector fields $X, Y$ on $M$;

(3) $\nabla_{X}(Y \circ Z)-\nabla_{Y}(X \circ Z)=\nabla_{X}^{*}(Y \circ Z)-\nabla_{Y}^{*}(X \circ Z)$ for all local vector fields $X, Y, Z$ on $M$.

Remark 2.3. Note that the condition $\mathcal{L}_{E} \mathrm{O}=0$ in these two definitions implies that $\mathcal{L}_{E} e=-e$.

The connections $\nabla$ and $\nabla^{*}$ are called the natural connection and the dual connection, respectively. The dual connection is defined at the points where the operator Eo is invertible. Moreover, from the above conditions it follows that it is uniquely determined in terms of the natural connection, the dual product, and the Euler vector field via the following expression:

$$
\Gamma_{i j}^{* k}=\Gamma_{i j}^{k}-c_{j i}^{* l} \nabla_{l} E^{k} .
$$

Conversely, the natural connection is uniquely determined in terms of the dual connection, the product, and the unit vector field via:

$$
\Gamma_{i j}^{k}=\Gamma_{i j}^{* k}-c_{j i}^{l} \nabla_{l}^{*} e^{k} .
$$

Compatibility of the dual connection with the dual product is a consequence of the other axioms (see AL19] for details) and flatness of the dual connection is equivalent to linearity of the Euler vector field (see [AL17] for the semisimple case and [KMS18] for the general case) $\nabla \nabla E=0$. Therefore, the structure of a bi-flat F-manifold is equivalent to the structure of a homogeneous flat F-manifold with invertible Euler vector field.

2.2. From homogeneous Riemannian F-manifolds to homogeneous flat F-manifolds. The construction of Theorem 1.8 produces a homogeneous flat F-manifold provided that we add some further conditions on a Riemannian F-manifold.

Definition 2.4. A homogeneous (pseudo-)Riemannian F-manifold is a (pseudo-)Riemannian F-manifold equipped with a distinguished vector field E, called the Euler vector field, such that the following conditions are satisfied:

$$
\mathcal{L}_{E} \mathrm{O}=\mathrm{\circ}, \quad \mathcal{L}_{E} g=D g,
$$

where $D$ is a constant. 
We can now state the main result of this section.

Theorem 2.5. Let $(M, \circ, g, e, E)$ be a homogeneous Riemannian F-manifold with Killing unit vector field. Then the data $(M, \circ, \nabla, e, E)$, where $\nabla$ is given by Theorem 1.8 , defines a homogeneous flat F-manifold.

Proof. We only need to prove that $\nabla \nabla E=0$. Recall that

$$
\Gamma_{j k}^{h}=\widetilde{\Gamma}_{j k}^{h}+b_{j k}^{h},
$$

where $\Gamma_{j k}^{h}$ are the Christoffel symbols of the connection $\nabla, \widetilde{\Gamma}_{j k}^{h}$ are the Christoffel symbols of the Levi-Civita connection of the metric $g$, and $b_{j k}^{h}=-\frac{1}{2} g^{s h} c_{k j}^{l} d \theta_{l s}$. Using the flatness of $\nabla$ we obtain

$$
(\nabla \nabla E)_{k j}^{i}=\partial_{k} \partial_{j} E^{i}+\Gamma_{j l}^{i} \partial_{k} E^{l}+\Gamma_{k m}^{i} \partial_{j} E^{m}-\Gamma_{k j}^{m} \partial_{m} E^{i}+E\left(\Gamma_{k j}^{i}\right) .
$$

From the homogeneity of the metric it follows that

$$
\begin{aligned}
& E\left(g^{i j}\right)=-D g^{i j}+g^{i s} \partial_{s} E^{j}+g^{s j} \partial_{s} E^{i}, \\
& E\left(g_{i j}\right)=D g_{i j}-g_{i s} \partial_{j} E^{s}-g_{s j} \partial_{i} E^{s} .
\end{aligned}
$$

Using these facts, after an elementary but long computation, one gets

$$
E\left(\widetilde{\Gamma}_{j k}^{i}\right)=\widetilde{\Gamma}_{j k}^{s} \partial_{s} E^{i}-\widetilde{\Gamma}_{j s}^{i} \partial_{k} E^{s}-\widetilde{\Gamma}_{s k}^{i} \partial_{j} E^{s}-\partial_{j} \partial_{k} E^{i} .
$$

Since $\mathcal{L}_{E} g=D g$ and $\mathcal{L}_{E} e=-e$, we have $\mathcal{L}_{E} \theta=(D-1) \theta$ and $\mathcal{L}_{E} d \theta=(D-1) d \theta$. Using also that $\mathcal{L}_{E} \mathrm{O}=\circ$ we conclude that $\mathcal{L}_{E} b_{j k}^{h}=0$ and, therefore,

$$
E\left(b_{j k}^{i}\right)=b_{j k}^{s} \partial_{s} E^{i}-b_{j s}^{i} \partial_{k} E^{s}-b_{s k}^{i} \partial_{j} E^{s} .
$$

Combining the above relations we get

$$
E\left(\Gamma_{j k}^{i}\right)=\Gamma_{j k}^{s} \partial_{s} E^{i}-\Gamma_{j s}^{i} \partial_{k} E^{s}-\Gamma_{s k}^{i} \partial_{j} E^{s}-\partial_{j} \partial_{k} E^{i}
$$

and substituting this on the right-hand side of (2.1) we get $\nabla \nabla E=0$.

\section{Riemannian F-Manifolds with Flat nORMal BUndLe}

A particular class of Riemannian F-manifolds consists of F-manifolds equipped with an invariant (pseudo-)metric $g$ satisfying condition (1.5) together with

$$
R_{k h}^{i j}=\sum_{\alpha=1}^{N} \varepsilon_{\alpha}\left(c_{k l}^{j} c_{h m}^{i}-c_{k l}^{i} c_{h m}^{j}\right) X_{(\alpha)}^{l} X_{(\alpha)}^{m}, \quad \varepsilon_{\alpha}= \pm 1,
$$

where $R_{k h}^{i j}=g^{i s} R_{s k h}^{j}$ are the components of the Riemann curvature tensor of the metric $g$ and the vector fields $X_{(\alpha)}$ satisfy the conditions

$$
c_{j l}^{i} \nabla_{k} X_{(\alpha)}^{l}=c_{k l}^{i} \nabla_{j} X_{(\alpha)}^{l},
$$

(the fact that relation (3.1) implies condition (1.4) is a simple direct computation). Here $\nabla$ is the flat connection given by Theorem 1.8, Let us call such Riemannian F-manifolds Riemannian F-manifolds with flat normal bundle.

Given a Riemannian F-manifold, it is easy to check that the affinors $W_{(\alpha)}:=X_{(\alpha)} \circ$ satisfy the conditions

$$
\begin{aligned}
& R_{k h}^{i j}=\sum_{\alpha=1}^{N} \varepsilon_{\alpha}\left(\left(W_{(\alpha)}\right)_{k}^{j}\left(W_{(\alpha)}\right)_{h}^{i}-\left(W_{(\alpha)}\right)_{k}^{i}\left(W_{(\alpha)}\right)_{h}^{j}\right), \\
& {\left[W_{(\alpha)}, W_{\left(\alpha^{\prime}\right)}\right]=0} \\
& g_{i k}\left(W_{(\alpha)}\right)_{j}^{k}=g_{j k}\left(W_{(\alpha)}\right)_{i}^{k}, \\
& \widetilde{\nabla}_{k}\left(W_{(\alpha)}\right)_{j}^{i}=\widetilde{\nabla}_{j}\left(W_{(\alpha)}\right)_{k}^{i},
\end{aligned}
$$


where $\widetilde{\nabla}$ is the Levi-Civita connection associated to the metric $g$. Equations (3.3) 3.4 3.5.3.6) can be interpreted as the Gauss-Peterson-Mainardi-Codazzi equations for an $n$-dimensional submanifold with flat normal connection embedded in an $(n+N)$-dimensional (pseudo-)Euclidean space. The (pseudo-)metric $g$ can be interpreted as the induced metric and the affinors $W_{(\alpha)}$ as Weingarten operators.

3.1. Riemannian F-manifolds with flat normal bundle and Hamiltonian operators. Conditions (3.3) 3.4 3.5, 3.6) also appear in the Hamiltonian formalism for systems of hydrodynamic type.

Theorem 3.1. Fer91] If $\operatorname{det}\left(g^{i j}\right) \neq 0$, then the operator

$$
P^{i j}=g^{i j} \frac{d}{d x}-g^{i s} \Gamma_{s k}^{j} u_{x}^{k}+\sum_{\alpha=1}^{N} \varepsilon_{\alpha}\left(W_{(\alpha)}\right)_{k}^{i} u_{x}^{k}\left(\frac{d}{d x}\right)^{-1}\left(W_{(\alpha)}\right)_{h}^{j} u_{x}^{h}, \quad \varepsilon_{\alpha}= \pm 1,
$$

defines a Hamiltonian operator if and only if $\left(g^{i j}\right)$ defines a pseudo-Riemannian metric, the coefficients $\Gamma_{s k}^{j}$ are the Christoffel symbols of the associated Levi-Civita connection $\nabla$, and the affinors $W_{(\alpha)}$ satisfy conditions (3.3, 3.4, 3.5,3.6).

We thus see that any Riemannian F-manifold with flat normal bundle gives, via this theorem, a non-local Hamiltonian operator.

Ferapontov also considered the limiting case where the index $\alpha$ takes values in infinite set (even continuous, with the sum replaced by an integral) and conjectured that any integrable diagonalizable quasilinear system of PDEs (semi-Hamiltonian system) is indeed Hamiltonian w.r.t. a suitable Poisson bracket of this class. This conjecture has been verified in the case of arbitrary n-component reductions of dKP [GLR09] and dispersionless 2D Toda hierarchy CLR10. In both cases the Riemann tensor of the generic reduction admits an integral representation that in some special cases reduces to a finite sum of residues at some marked points. In the case of semisimple Riemannian F-manifolds condition (1.4) ensures that all flows of the associated principal hierarchy are semi-Hamiltonian. Thus, validity of the Ferapontov conjecture in this setting would imply the possibility of writing the Riemann tensor associated with the invariant metric $g$ of a Riemannian F-manifold in terms of the solutions of system (3.2) with the finite sum in (3.1) possibly replaced by an integral.

\subsection{An example with a special Lauricella bi-flat F-manifold.}

Proposition 3.2. ([ [L13, Section 7], [Lor14, Section 5]) The connection $\nabla$ defined by the Christoffel symbols

$$
\Gamma_{j k}^{i}:=0, \quad \Gamma_{j j}^{i}:=-\Gamma_{i j}^{i}, \quad \Gamma_{i j}^{i}:=\frac{\epsilon_{j}}{u^{i}-u^{j}}, \quad \Gamma_{i i}^{i}:=-\sum_{l \neq i} \Gamma_{l i}^{i}, \quad i \neq j \neq k \neq i,
$$

the dual connection $\nabla^{*}$ defined by the Christoffel symbols

$$
\Gamma_{j k}^{* i}:=0, \quad \Gamma_{j j}^{* i}:=-\frac{u^{i}}{u^{j}} \Gamma_{i j}^{* i}, \quad \Gamma_{i j}^{* i}:=\frac{\epsilon_{j}}{u^{i}-u^{j}}, \quad \Gamma_{i i}^{* i}:=-\sum_{l \neq i} \frac{u^{l}}{u^{i}} \Gamma_{l i}^{* i}-\frac{1}{u^{i}}, \quad i \neq j \neq k \neq i,
$$

the products $c_{j k}^{i}:=\delta_{j}^{i} \delta_{k}^{i}$ and $c_{j k}^{* i}:=\frac{1}{u^{i}} \delta_{j}^{i} \delta_{k}^{i}$ and the vector fields e $:=\sum_{k=1}^{n} \partial_{k}$ and $E:=\sum_{k=1}^{n} u^{k} \partial_{k}$ define a bi-flat semisimple F-manifold structure for any choice of $\epsilon_{1}, \ldots, \epsilon_{n}$.

These examples are related to the theory of Lauricella functions [Lau1893, Lauricella connections, and Lauricella manifolds [CHL05, LO007] and for this reason are called the Lauricella bi-flat structures [AL19.

We focus on the case $\epsilon_{i}=-1, i=1, \ldots, n$. This is related to the system of chromatography equations [Fer91]. The invariant metric in this case has non-vanishing components

$$
g_{i i}=c_{i} \prod_{l \neq i}\left(u^{l}-u^{i}\right)^{2}, \quad i=1, \ldots, n,
$$


where $c_{1}, \ldots, c_{n}$ are arbitrary nonzero constants. It was observed in [Fer91, Example 5] that the associated Riemann tensor admits the following quadratic expansion:

$$
R_{i j}^{i j}=-\sum_{\alpha=1}^{n} X_{(\alpha)}^{i} X_{(\alpha)}^{j}, \quad i \neq j,
$$

where

$$
X_{(\alpha)}^{i}=\partial_{i}\left(\frac{1}{\sqrt{c_{\alpha}} \prod_{l \neq \alpha}\left(u^{l}-u^{\alpha}\right)}\right) .
$$

This implies that condition (3.1) is satisfied.

Proposition 3.3. The vector fields $X_{(\alpha)}^{i}, \alpha=1, \ldots, n$ of this specific example are covariantly constant w.r.t. the flat connection $\nabla$ with $\epsilon_{i}=-1, i=1, \ldots, n$. The rank of the $n \times n$ matrix whose columns are the vector fields $X_{(\alpha)}$ is $n-1$.

Proof. The proof is by a straightforward computation.

Therefore, the Lauricella bi-flat F-manifolds with $\epsilon_{i}=-1$ are Riemannian F-manifolds with flat normal bundle.

3.3. An example with the Lobachevsky hyperbolic half-plane. The Riemann tensor of the Lobachevsky hyperbolic half-plane from Section 1.3 is given by $R_{12}^{12}=1$ and thus condition (3.1) is satisfied with $\alpha=1, \varepsilon_{1}=-1$, and $X_{(1)}=e$. Since $\nabla e=0$ condition (3.2) is also satisfied. Therefore, the Lobachevsky hyperbolic half-plane is a Riemannian F-manifold with flat normal bundle. The associated non-local Hamiltonian operator given by Theorem 3.1 is

$$
\begin{aligned}
\left(P^{i j}\right)= & \left(\begin{array}{cc}
\frac{\left(u^{1}-u^{2}\right)^{2}}{2} & 0 \\
0 & \frac{\left(u^{1}-u^{2}\right)^{2}}{2}
\end{array}\right) \frac{d}{d x}+\left(\begin{array}{cc}
\frac{u^{1}-u^{2}}{2}\left(u_{x}^{1}-u_{x}^{2}\right) & \frac{u^{1}-u^{2}}{2}\left(u_{x}^{1}+u_{x}^{2}\right) \\
-\frac{u^{1}-u^{2}}{2}\left(u_{x}^{1}+u_{x}^{2}\right) & \frac{u^{1}-u^{2}}{2}\left(u_{x}^{1}-u_{x}^{2}\right)
\end{array}\right) \\
& -\left(\begin{array}{cc}
u_{x}^{1}\left(\frac{d}{d x}\right)^{-1} u_{x}^{1} & u_{x}^{1}\left(\frac{d}{d x}\right)^{-1} u_{x}^{2} \\
u_{x}^{2}\left(\frac{d}{d x}\right)^{-1} u_{x}^{1} & u_{x}^{2}\left(\frac{d}{d x}\right)^{-1} u_{x}^{2}
\end{array}\right) .
\end{aligned}
$$

\section{Flat homogeneous Riemannian F-manifolds}

Let $(M, \circ, g, e, E)$ be a semisimple homogeneous (pseudo-)Riemannian F-manifold with Killing unit vector field. In canonical coordinates the metric $g$ is diagonal. Let us introduce the Lamé coefficients $H_{i}:=\sqrt{g_{i i}}$ and the Ricci rotation coefficients $\beta_{i j}:=\frac{\partial_{j} H_{i}}{H_{j}}, i \neq j$. It is easy to check that they satisfy the following overdetermined system of PDEs:

$$
\begin{aligned}
\partial_{k} \beta_{i j} & =\beta_{i k} \beta_{k j}, & & i \neq j \neq k \neq i, \\
e\left(\beta_{i j}\right) & =0, & & i \neq j, \\
E\left(\beta_{i j}\right) & =-\beta_{i j}, & & i \neq j,
\end{aligned}
$$

where

$$
e=\sum_{i=1}^{n} \partial_{i}, \quad E=\sum_{i=1}^{n} u^{i} \partial_{i} .
$$

Given a solution of the above system, the Lamé coefficients of the metric $g$ are obtained solving the overdetermined system of PDEs

$$
\begin{aligned}
\partial_{j} H_{i} & =\beta_{i j} H_{j}, & i \neq j, \\
e\left(H_{i}\right) & =0, & \\
E\left(H_{i}\right) & =d H_{i}, &
\end{aligned}
$$

where $d$ is an eigenvalue of the matrix $V_{i j}:=\left(u^{j}-u^{i}\right) \beta_{i j}$ Dub96, AL13]. Note that then $\mathcal{L}_{E} g=D g$ with $D=2 d+2$. 
This system has been extensively studied in [AL13] where it has been shown that in the case $n=3$ it is equivalent to a 2-parameter family of Painlevé VI. Replacing equation (4.3) with the equation

$$
E\left(\beta_{i j}\right)=\left(d_{i}-d_{j}-1\right) \beta_{i j}, \quad i \neq j,
$$

and equation (4.6) with the equation

$$
E\left(H_{i}\right)=d_{i} H_{i},
$$

one gets the system for general semisimple bi-flat F-manifolds [Lor14]. In this case only the differences of the constants $d_{i}$ can be chosen arbitrarily, while $d_{1}$ must be an eigenvalue of the matrix $V_{i j}:=\left(u^{j}-u^{i}\right) \beta_{i j}-\left(d_{j}-d_{1}\right) \delta_{j}^{i}$. Comparing the system for generic semisimple bi-flat F-manifolds to the special case related to homogeneous Riemannian F-manifolds with Killing unit vector field it is clear that the first one reduces to the second one imposing the condition $d_{1}=d_{2}=\ldots=d_{n}$.

In this section we want to study solutions of the system $((4.1),(4.2),(4.3),(4.4),(4.5),(4.6))$ for which the resulting metric $g$ is flat. This amounts to consider the additional conditions

$$
\partial_{i} \beta_{j i}+\partial_{j} \beta_{i j}+\sum_{k \neq i, j} \beta_{i k} \beta_{j k}=0, \quad i \neq j .
$$

These additional conditions are automatically fulfilled if the rotation coefficients are symmetric. This is the special case corresponding to exact homogeneous flat pencils satisfying the Egorov property, which are equivalent to Dubrovin-Frobenius manifolds.

Using (4.2) and (4.3) we can reduce conditions (4.7) to a set of $\frac{n(n-1)}{2}$ algebraic constraints. Indeed, from (4.2) and (4.3) we get

$$
\partial_{j} \beta_{i j}=\frac{1}{u^{j}-u^{i}}\left\{\sum_{k \neq i, j}\left(u^{i}-u^{k}\right) \partial_{k} \beta_{i j}-\beta_{i j}\right\}, \quad i \neq j,
$$

and thus (4.7) becomes

$$
\sum_{k \neq i, j}\left[\left(u^{j}-u^{k}\right)(\Delta \beta)_{i k} \beta_{j k}+\left(u^{k}-u^{i}\right)(\Delta \beta)_{j k} \beta_{i k}\right]=(\Delta \beta)_{i j}, \quad i \neq j,
$$

where $(\Delta \beta)_{i j}:=\beta_{i j}-\beta_{j i}$. In the Egorov case $(\Delta \beta)_{i j}=0$ the above conditions are automatically satisfied. The Egorov conditions imply $\partial_{j}\left(H_{i}^{2}\right)=\partial_{i}\left(H_{j}^{2}\right)$ and thus (locally) the metrics with rotation coefficients $\beta_{i j}$ are potential in the coordinates $\left(u^{1}, \ldots, u^{n}\right)$. The Egorov conditions are not invariant with respect to transformations $u^{i} \rightarrow \tilde{u}^{i}=\varphi^{i}\left(u^{i}\right)$ preserving the diagonal form of the metric. Metrics that are potential for a suitable choice of $\tilde{u}^{i}$ satisfy the weaker conditions Dar10, Ego70

$$
\beta_{i j} \beta_{j k} \beta_{k i}=\beta_{j i} \beta_{i k} \beta_{k j}, \quad i \neq j \neq k \neq i .
$$

4.1. The three-dimensional case. The case $n=2$ is trivial. In this case condition (4.8) reduces to $(\Delta \beta)_{i j}=0$. Let us consider the case $n=3$. Following [AL13] we can reduce the system of partial differential equations (4.1,4.24.3) to a system of ODEs. Indeed, equations (4.2) and (4.3) tell us that the functions $\beta_{i j}$ are homogeneous functions of degree -1 which depend only on the differences of the coordinates. This means that we can write

$$
\begin{array}{ll}
\beta_{12}=\frac{1}{u^{2}-u^{1}} F_{12}\left(\frac{u^{3}-u^{1}}{u^{2}-u^{1}}\right), & \beta_{21}=\frac{1}{u^{2}-u^{1}} F_{21}\left(\frac{u^{3}-u^{1}}{u^{2}-u^{1}}\right), \\
\beta_{13}=\frac{1}{u^{3}-u^{1}} F_{13}\left(\frac{u^{3}-u^{1}}{u^{2}-u^{1}}\right), & \beta_{31}=\frac{1}{u^{3}-u^{1}} F_{31}\left(\frac{u^{3}-u^{1}}{u^{2}-u^{1}}\right), \\
\beta_{23}=\frac{1}{u^{3}-u^{2}} F_{23}\left(\frac{u^{3}-u^{1}}{u^{2}-u^{1}}\right), & \beta_{32}=\frac{1}{u^{3}-u^{2}} F_{32}\left(\frac{u^{3}-u^{1}}{u^{2}-u^{1}}\right) .
\end{array}
$$


Introducing the variable $z=\frac{u^{3}-u^{1}}{u^{2}-u^{1}}$ we get the system

$$
\begin{aligned}
\frac{d F_{12}}{d z} & =\frac{1}{z(z-1)} F_{13} F_{32}, & \frac{d F_{21}}{d z} & =\frac{1}{z(z-1)} F_{23} F_{31}, \\
\frac{d F_{13}}{d z} & =-\frac{1}{z-1} F_{12} F_{23}, & \frac{d F_{31}}{d z} & =-\frac{1}{z-1} F_{32} F_{21}, \\
\frac{d F_{23}}{d z} & =\frac{1}{z} F_{21} F_{13}, & \frac{d F_{32}}{d z} & =\frac{1}{z} F_{31} F_{12} .
\end{aligned}
$$

The above system admits two first integrals $I_{1}, I_{2}$ given by $\operatorname{det}(V+\lambda \mathrm{Id})=\lambda^{3}+\lambda I_{1}+I_{2}$ or, explicitly,

$$
I_{1}=F_{12} F_{21}+F_{13} F_{31}+F_{23} F_{32}, \quad I_{2}=F_{13} F_{32} F_{21}-F_{23} F_{31} F_{12},
$$

and

$$
V=\left[\begin{array}{ccc}
0 & F_{12} & F_{13} \\
-F_{21} & 0 & F_{23} \\
-F_{31} & -F_{32} & 0
\end{array}\right]
$$

The constraint (4.8) reads

$$
\begin{aligned}
& \left(u^{2}-u^{3}\right)(\Delta \beta)_{13} \beta_{23}+\left(u^{3}-u^{1}\right)(\Delta \beta)_{23} \beta_{13}-(\Delta \beta)_{12}=0, \\
& \left(u^{1}-u^{3}\right)(\Delta \beta)_{12} \beta_{31}+\left(u^{2}-u^{1}\right)(\Delta \beta)_{13} \beta_{21}-(\Delta \beta)_{23}=0 \\
& \left(u^{2}-u^{1}\right)(\Delta \beta)_{23} \beta_{12}+\left(u^{2}-u^{3}\right)(\Delta \beta)_{12} \beta_{32}+(\Delta \beta)_{13}=0
\end{aligned}
$$

or, in terms of the functions $F_{i j}$,

$$
\begin{aligned}
& I_{3}=\left(z^{2}-z\right)(\Delta F)_{12}+(z-1) F_{23}(\Delta F)_{13}-z F_{13}\left(\Delta F_{23}\right)=0, \\
& I_{4}=\left(z^{2}-z\right) F_{31}(\Delta F)_{12}+(1-z) F_{21}(\Delta F)_{13}+z(\Delta F)_{23}=0, \\
& I_{5}=\left(z^{2}-z\right) F_{32}(\Delta F)_{12}+(1-z)(\Delta F)_{13}-z F_{12}(\Delta F)_{23}=0,
\end{aligned}
$$

where $(\Delta F)_{i j}:=F_{i j}-F_{j i}$. This can also be written in the matrix form as

$$
W\left[\begin{array}{l}
(\Delta F)_{12} \\
(\Delta F)_{13} \\
(\Delta F)_{23}
\end{array}\right]=\left[\begin{array}{l}
0 \\
0 \\
0
\end{array}\right], \quad \text { where } W=\left[\begin{array}{ccc}
z^{2}-z & (z-1) F_{23} & -z F_{13} \\
\left(z^{2}-z\right) F_{31} & (1-z) F_{21} & z \\
\left(z^{2}-z\right) F_{32} & 1-z & -z F_{12}
\end{array}\right] .
$$

It is easy to check that

$$
\operatorname{det} W=z^{2}(z-1)^{2}\left(I_{1}-I_{2}+1\right) .
$$

Here we have to distinguish two cases:

(1) The Egorov case: $(\Delta \beta)_{i j}=0$ for all $i \neq j$. In this case the constraints $I_{3}, I_{4}, I_{5}$ are automatically satisfied.

(2) The non-Egorov case: $(\Delta \beta)_{i j} \neq 0$ for some $i \neq j$. In this case the determinant of the matrix of the system must vanish. As a consequence, the values of the two first integrals cannot be chosen independently:

$$
I_{1}=I_{2}-1
$$

Using this relation and the fact that the vector $\left((\Delta F)_{12},(\Delta F)_{13},(\Delta F)_{23}\right)^{t}$ belongs to the kernel of $W$ it is possible to prove that the constraints $I_{3}=0, I_{4}=0, I_{5}=0$ are compatible with the system of ODEs for the functions $F_{i j}$. In other words, this system can be reduced to the set defined by the algebraic system $I_{1}=q-1, I_{2}=$ $q, I_{3}=0, I_{4}=0, I_{5}=0$, where $q$ is a constant. Since the functions $I_{i}$ are, at a generic point, functionally independent, it would be reasonable to expect that the system for the functions $F_{i j}$ reduces to a single first order ODE on this set. Remarkably, this is not the case due to a jump in the rank of the Jacobian of the functions $I_{i}$ on this set. 
4.1.1. An example: the case $q=0$. Let us consider for instance the metrics satisfying the potentiality condition (4.9). In this case $q=0$ and the solution of the algebraic system $I_{1}=$ $-1, I_{2}=0, I_{3}=0, I_{4}=0, I_{5}=0$ (obtained using the computational software Maple) is given by

$$
\begin{aligned}
F_{12}= & \frac{(z-1)\left(F_{21}^{3} F_{31}^{2}+F_{21} F_{31}^{4}-F_{21}^{3}+F_{21} F_{31}^{2}\right)-F_{21}\left(F_{21}^{2}+F_{31}^{2}\right)+\delta\left((z-1) F_{21}^{2} F_{31}+F_{31}^{3}\right)}{z\left(F_{21}^{2}+F_{31}^{2}\right)^{2}}, \\
F_{32}= & \frac{-F_{31}(z-1)\left(-F_{21}^{5}+F_{21}^{3} F_{31}^{2}+2 F_{21} F_{31}^{4}-F_{21}^{3}+3 F_{21} F_{31}^{2}\right)}{z\left(-F_{21}^{4}-F_{21}^{2} F_{31}^{2}-F_{21}^{2}+F_{31}^{2}\right)\left(F_{21}^{2}+F_{31}^{2}\right)} \\
& +\frac{-F_{31}(z-1) \delta\left(F_{21}^{4} F_{31}+F_{21}^{2} F_{31}^{3}+3 F_{21}^{2} F_{31}-F_{31}^{3}\right)}{z\left(-F_{21}^{4}-F_{21}^{2} F_{31}^{2}+2 \delta F_{21} F_{31}-F_{21}^{2}+F_{31}^{2}\right)\left(F_{21}^{2}+F_{31}^{2}\right)}, \\
F_{23}= & \frac{(z-1)\left(3 F_{21}^{4} F_{31}+3 F_{21}^{2} F_{31}^{3}+3 F_{21}^{2} F_{31}-F_{31}^{3}\right) F_{21}}{\left(-F_{21}^{4}-F_{21}^{2} F_{31}^{2}+2 \delta F_{21} F_{31}-F_{21}^{2}+F_{31}^{2}\right)\left(F_{21}^{2}+F_{31}^{2}\right)} \\
& +\frac{(z-1) \delta F_{21}\left(F_{21}^{5}+F_{21}^{3} F_{31}^{2}+F_{21}^{3}-3 F_{21} F_{31}^{2}\right) F_{21}}{\left(-F_{21}^{4}-F_{21}^{2} F_{31}^{2}+2 \delta F_{21} F_{31}-F_{21}^{2}+F_{31}^{2}\right)\left(F_{21}^{2}+F_{31}^{2}\right)},
\end{aligned}
$$

where $\delta=\sqrt{-F_{31}^{2}-F_{21}^{2}-1}$ and the function $F_{13}$ is obtained substituting the previous expressions in

$$
F_{13}=\frac{F_{31} F_{23} F_{12}}{F_{21} F_{32}}
$$

Moreover, on this set the Jacobian of the functions $I_{i}$ has rank 4 .

The reduced system is

$\begin{aligned} \frac{d F_{21}}{d z} & =\frac{F_{21} F_{31}\left[3 F_{21}^{4} F_{31}+3 F_{21}^{2} F_{31}^{3}+3 F_{21}^{2} F_{31}-F_{31}^{3}+\delta\left(F_{21}^{5}+F_{21}^{3} F_{31}^{2}+F_{21}^{3}-3 F_{21} F_{31}^{2}\right)\right]}{z\left(-F_{21}^{4}-F_{21}^{2} F_{31}^{2}+2 \delta F_{21} F_{31}-F_{21}^{2}+F_{31}^{2}\right)\left(F_{21}^{2}+F_{31}^{2}\right)}, \\ \frac{d F_{31}}{d z} & =\frac{\left[F_{21}^{5}-F_{21}^{3} F_{31}^{2}-2 F_{21} F_{31}^{4}+F_{21}^{3}-3 F_{21} F_{31}^{2}+\delta\left(-F_{21}^{4} F_{31}-F_{21}^{2} F_{31}^{3}-3 F_{21}^{2} F_{31}+F_{31}^{3}\right)\right] F_{31} F_{21}}{z\left(F_{21}^{4}+F_{21}^{2} F_{31}^{2}-2 \delta F_{21} F_{31}+F_{21}^{2}-F_{31}^{2}\right)\left(F_{21}^{2}+F_{31}^{2}\right)} .\end{aligned}$

The general solution is

$$
F_{21}=-\frac{1}{a z+b}, \quad F_{31}=-\frac{a z}{(a z+b) \sqrt{-b^{2}-1}} .
$$

Starting from this solution we get

$$
\begin{array}{ll}
F_{21}=\frac{\left(u^{2}-u^{1}\right)}{\left(a u^{1}-a u^{3}+b u^{1}-b u^{2}\right)}, & F_{31}=-\frac{a\left(u^{1}-u^{3}\right)}{\left(a u^{1}-a u^{3}+b u^{1}-b u^{2}\right) \sqrt{-b^{2}-1}}, \\
F_{12}=\frac{b(a+b)\left(u^{1}-u^{2}\right)}{\left(a u^{1}-a u^{3}+b u^{1}-b u^{2}\right)}, & F_{13}=\frac{\left(u^{1}-u^{3}\right)(a+b) \sqrt{-b^{2}-1}}{\left(a u^{1}-a u^{3}+b u^{1}-b u^{2}\right)}, \\
F_{32}=\frac{\left(u^{3}-u^{2}\right) a b}{\sqrt{-b^{2}-1}\left(a u^{1}-a u^{3}+b u^{1}-b u^{2}\right)}, & F_{23}=\frac{\left(u^{3}-u^{2}\right) \sqrt{-b^{2}-1}}{\left(a u^{1}-a u^{3}+b u^{1}-b u^{2}\right)} .
\end{array}
$$

The matrix $V$ in this case has eigenvalues $1,0,-1$. This means that if $d=1,0,-1$, then the overdetermined system for the Lamé coefficients (4.4 4.5.4.6) admits solutions. For $d=-1$ we get

$$
\begin{aligned}
& H_{1}=\frac{1}{(a+b) u^{1}-a u^{3}-b u^{2}} \\
& H_{2}=-\frac{1}{(a+b)\left(\left(u^{1}-u^{2}\right) b+a\left(u^{1}-u^{3}\right)\right)} \\
& H_{3}=-\frac{a}{\sqrt{-b^{2}-1}(a+b)\left(\left(u^{1}-u^{2}\right) b+a\left(u^{1}-u^{3}\right)\right)} .
\end{aligned}
$$


For $d=0$ we get

$$
\begin{aligned}
& H_{1}=\frac{\left(u^{2}-u^{3}\right)}{\left((a+b) u^{1}-a u^{3}-b u^{2}\right)}, \\
& H_{2}=\frac{\left(u^{3}-u^{1}\right)}{\left(\left(u^{1}-u^{2}\right) b+a\left(u^{1}-u^{3}\right)\right) b} \\
& H_{3}=\frac{\left(u^{1}-u^{2}\right)}{\left((a+b) u^{1}-a u^{3}-b u^{2}\right) \sqrt{-b^{2}-1}},
\end{aligned}
$$

and for $d=1$ we get

$$
\begin{aligned}
& H_{1}=-\frac{\left((a+b)\left(u^{1}\right)^{2}-2\left(a u^{3}+b u^{2}\right) u^{1}+a\left(u^{3}\right)^{2}+b\left(u^{2}\right)^{2}\right)}{\left((a+b) u^{1}-a u^{3}-b u^{2}\right)} \\
& H_{2}=-\frac{\left((a+b)\left(u^{1}\right)^{2}-2 u^{2}(a+b) u^{1}+\left(2 u^{2} u^{3}-\left(u^{3}\right)^{2}\right) a+b\left(u^{2}\right)^{2}\right)}{\left((a+b) u^{1}-a u^{3}-b u^{2}\right)(a+b)} \\
& H_{3}=-\frac{\left((a+b)\left(u^{1}\right)^{2}-2 u^{3}(a+b) u^{1}+a\left(u^{3}\right)^{2}-b u^{2}\left(u^{2}-2 u^{3}\right)\right) a}{\sqrt{-b^{2}-1}\left((a+b) u^{1}-a u^{3}-b u^{2}\right)(a+b)}
\end{aligned}
$$

Solutions corresponding to generic values of $q$ can be treated in a similar way but the computations are much more involved.

Remark 4.1. In the case $q=0$ the rotation coefficients satisfy the potentiality condition (4.9). This means that there exist coordinates $\left(\tilde{u}^{1}, \ldots, \tilde{u}^{n}\right)$ related to canonical coordinates $\left(u^{1}, \ldots, u^{n}\right)$ by $\tilde{u}^{i}=\varphi^{i}\left(u^{i}\right)$ (for some functions $\varphi^{i}$ ) reducing the metric to the potential form. In other words this is the closest case to Dubrovin-Frobenius manifolds.

\section{EXACT HOMOGENEOUS FLAT PENCILS OF METRICS}

Definition 5.1. A pair of contravariant metrics $\left(g_{1}, g_{2}\right)$ defines a flat pencil of metrics $g_{2}-\lambda g_{1}$ if and only if the following conditions are satisfied:

- the metric $g_{2}-\lambda g_{1}$ is flat for any $\lambda$;

- the contravariant Christoffel symbols $\Gamma_{(\lambda) k}^{i j}$ of the pencil are the pencil of the contravariant Christoffel symbols:

$$
\Gamma_{(\lambda) k}^{i j}=\Gamma_{(2) k}^{i j}-\lambda \Gamma_{(1) k}^{i j} .
$$

Flat pencils of contravariant metrics play a crucial role in the theory of Dubrovin-Frobenius manifolds. Dubrovin proved that any Dubrovin-Frobenius manifold defines a flat pencil of contravariant metrics ([Dub96]). In this case $g_{1}$ coincides with the inverse of the covariant invariant metric $\eta$ of the Dubrovin-Frobenius manifold and $g_{2}$ is the intersection form defined by

$$
g_{2}^{i j}:=\eta^{i l} c_{l k}^{j} E^{k}
$$

where $c_{l k}^{j}$ are the structure constants of the product and $E^{k}$ are the components of the Euler vector field. Vice versa, Dubrovin showed how to construct a Dubrovin-Frobenius manifold starting from a flat pencil of metrics satisfying the following three additional properties:

- Exactness: there exists a vector field $e$ such that

$$
\mathcal{L}_{e} g_{2}=g_{1}, \quad \mathcal{L}_{e} g_{1}=0 .
$$

- Homogeneity:

$$
\mathcal{L}_{E} g_{2}=(d-1) g_{2},
$$

where $E^{i}:=g_{2}^{i l}\left(g_{1}\right)_{l j} e^{j}$

- Egorov property: locally there exists a function $\tau$ such that

$$
e^{i}=g_{1}^{i s} \partial_{s} \tau, \quad E^{i}=g_{2}^{i s} \partial_{s} \tau .
$$


Remark 5.2. Exactness implies that $[e, E]=e$ and combining this with the homogeneity condition we get

$$
\mathcal{L}_{E} g_{1}=\mathcal{L}_{E} \mathcal{L}_{e} g_{2}=\mathcal{L}_{e} \mathcal{L}_{E} g_{2}-\mathcal{L}_{[E, e]} g_{2}=(d-2) g_{1} .
$$

Remark 5.3. In the case of Dubrovin-Frobenius manifolds the vector fields e and E coincide with the unit vector field and the Euler vector field, respectively.

\subsection{From flat pencils of metrics to homogeneous Riemannian F-manifolds.}

Theorem 5.4. Let $g_{\lambda}=g-\lambda \eta$ be an exact homogeneous flat pencil of metrics such that the operator $R$ defined by

$$
R_{j}^{i}:=\nabla_{(1) j} E^{i}-\nabla_{(2) j} E^{i}
$$

is invertible, where $\nabla_{(1)}$ and $\nabla_{(2)}$ are the Levi-Civita connections corresponding to the metrics $\eta$ and $g$, respectively, and we recall that $E^{i}=g^{i l} \eta_{l j} e^{j}$. Then the data $(\circ, \eta, e, E)$, where the product $\circ$ is defined by the structure constants

$$
c_{h k}^{j}:=L_{h}^{s}\left(\Gamma_{s k}^{(1) l}-\Gamma_{s k}^{(2) l}\right)\left(R^{-1}\right)_{l}^{j}, \quad L_{h}^{s}:=g^{s m} \eta_{m h},
$$

defines a homogeneous flat (pseudo-)Riemannian F-manifold with Killing unit vector field.

Proof. To prove the theorem we need some results from [Dub98]. More precisely, let us introduce the tensor field

$$
\Delta_{m}^{j k}:=L_{m}^{s} \eta^{j t}\left(\Gamma_{s t}^{(1) k}-\Gamma_{s t}^{(2) k}\right) .
$$

The following identities hold true [Dub98, Lemmas 2.2 and 2.6]:

$$
\begin{aligned}
\eta^{i s} \Delta_{s}^{j k} & =\eta^{j s} \Delta_{s}^{i k}, \\
g^{i s} \Delta_{s}^{j k} & =g^{j s} \Delta_{s}^{i k} \\
\Delta_{s}^{i j} \Delta_{l}^{s k} & =\Delta_{s}^{i k} \Delta_{l}^{s j} \\
\mathcal{L}_{E} \Delta_{i}^{j k} & =(d-1) \Delta_{i}^{j k} .
\end{aligned}
$$

Note that from the first two identities it follows that

$$
\begin{aligned}
\eta_{h s} \Delta_{m}^{s k} & =\eta_{m s} \Delta_{h}^{s k}, \\
g_{h s} \Delta_{m}^{s k} & =g_{m s} \Delta_{h}^{s k} .
\end{aligned}
$$

Using the tensor $\Delta$, the tensors $R$ and $c$ can be expressed as follows:

$$
\begin{aligned}
& R_{s}^{m}=\left(\Gamma_{s l}^{(1) m}-\Gamma_{s l}^{(2) m}\right) E^{l}=g_{s r} \eta^{r q} \Delta_{q}^{p m} \eta_{p l} E^{l} \stackrel{\sqrt{5.30}}{=} g_{s p} \Delta_{l}^{p m} E^{l}, \\
& c_{h k}^{j}=\Delta_{h}^{m l} \eta_{m k}\left(R^{-1}\right)_{l}^{j} .
\end{aligned}
$$

Lemma 5.5. The following identity holds true:

$$
\Delta_{k}^{t l}\left(R^{-1}\right)_{l}^{s}=\Delta_{k}^{s l}\left(R^{-1}\right)_{l}^{t}
$$

Proof. Equivalently, we have to prove that $\Delta_{k}^{s h} R_{s}^{m}=\Delta_{k}^{s m} R_{s}^{h}$, for which we compute

$$
\Delta_{k}^{s h} R_{s}^{m}=\Delta_{k}^{s h} g_{s p} \Delta_{l}^{p m} E^{l} \stackrel{(5.4)}{=} \Delta_{p}^{s h} g_{s k} \Delta_{l}^{p m} E^{l} \stackrel{(5.1)}{=} \Delta_{p}^{s m} g_{s k} \Delta_{l}^{p h} E^{l} \stackrel{\sqrt{5.4}}{=} \Delta_{k}^{s m} g_{s p} \Delta_{l}^{p h} E^{l}=\Delta_{k}^{s m} R_{s}^{h},
$$

as required.

In order to prove the theorem, we need to prove the following:

- The product is commutative:

$$
c_{h k}^{j}=\Delta_{h}^{m l} \eta_{m k}\left(R^{-1}\right)_{l}^{j} \stackrel{(5.3)}{=} \Delta_{k}^{m l} \eta_{m h}\left(R^{-1}\right)_{l}^{j}=c_{k h}^{j} .
$$


- The product is associative:

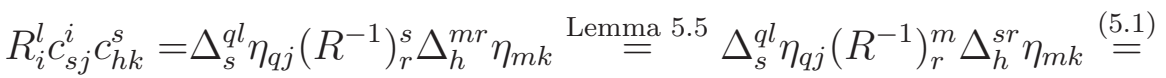

$$
\begin{aligned}
& =\Delta_{s}^{q r} \eta_{q j}\left(R^{-1}\right)_{r}^{m} \Delta_{h}^{s l} \eta_{m k} \stackrel{\text { Lemma 5.5 }}{=} \Delta_{s}^{m r} \eta_{q j}\left(R^{-1}\right)_{r}^{q} \Delta_{h}^{s l} \eta_{m k} \stackrel{5.1]}{=}
\end{aligned}
$$

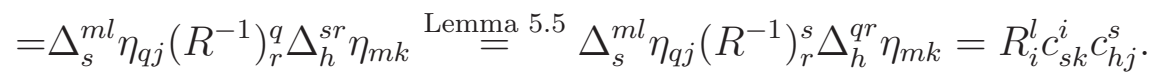

- The vector field $e$ is the unit of the product:

$$
c_{h k}^{j} e^{h}=L_{h}^{s}\left(\Gamma_{s k}^{(1) l}-\Gamma_{s k}^{(2) l}\right)\left(R^{-1}\right)_{l}^{j} e^{h}=E^{s}\left(\Gamma_{s k}^{(1) l}-\Gamma_{s k}^{(2) l}\right)\left(R^{-1}\right)_{l}^{j}=R_{k}^{l}\left(R^{-1}\right)_{l}^{j}=\delta_{k}^{j} .
$$

- The metric $\eta$ is invariant with respect to the product:

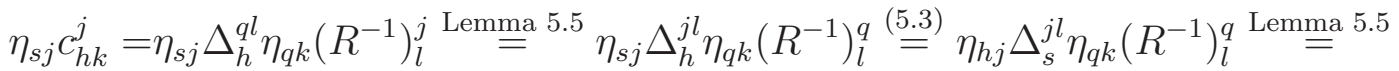

$$
\begin{aligned}
& =\eta_{h j} \Delta_{s}^{q l} \eta_{q k}\left(R^{-1}\right)_{l}^{j}=\eta_{h j} c_{s k}^{j} \text {. }
\end{aligned}
$$

- $\mathcal{L}_{E} \mathrm{O}=$ o. This is true, because equation (5.2) first implies that $\mathcal{L}_{E} R_{j}^{i}=0$, and then using (5.5) $)$ it gives that $\mathcal{L}_{E} c_{h k}^{j}=c_{h k}^{j}$, since $\mathcal{L}_{E} \eta_{i j}=(2-d) \eta_{i j}$.

Remark 5.6. It is easy to check that the affinor L coincides with the operator of multiplication by the Euler vector field. Indeed we have

$$
c_{h k}^{j} E^{h}=g^{m s} \Delta_{s h}^{l} E^{h} \eta_{m k}\left(R^{-1}\right)_{l}^{j}=g^{m s} R_{s}^{l} \eta_{m k}\left(R^{-1}\right)_{l}^{j}=g^{m s} \eta_{m k} \delta_{s}^{j}=g^{m j} \eta_{m k}=L_{k}^{j} .
$$

Remark 5.7. It is is easy to prove that

$$
R_{j}^{i}=\frac{d-1}{2} \delta_{j}^{i}+\nabla_{(1) j} E^{i}+\frac{1}{2} g^{i s} d \theta_{s j} .
$$

Indeed,

$$
\begin{aligned}
\nabla_{(2) i} E^{k} & =\partial_{i} E^{k}+\frac{1}{2} g^{k s}\left(\partial_{i} g_{j s} E^{j}+E^{j} \partial_{j} g_{s i}-\partial_{s} g_{i j} E^{j}\right)= \\
& =\partial_{i} E^{k}+\frac{1}{2} g^{k s}\left(\partial_{i} g_{j s} E^{j}-g_{s j} \partial_{i} E^{j}-g_{i j} \partial_{s} E^{j}+(1-d) g_{s i}-\partial_{s} g_{i j} E^{j}\right)= \\
& =\partial_{i} E^{k}+\frac{1}{2}\left(g^{k s} \partial_{i} g_{j s} E^{j}-\partial_{i} E^{k}-g^{k s} \partial_{s}\left(g_{i j} E^{j}\right)+(1-d) \delta_{i}^{k}\right)= \\
& =\frac{1}{2} \partial_{i} E^{k}+\frac{1}{2}\left(g^{k s} \partial_{i} g_{j s} E^{j}-g^{k s} \partial_{s}\left(g_{i j} E^{j}\right)\right)+\frac{1-d}{2} \delta_{i}^{k}= \\
& =\frac{1}{2} \partial_{i} E^{k}+\frac{1}{2}\left(g^{k s} \partial_{i} g_{j s} E^{j}-g^{k s} \partial_{s} \theta_{i}\right)+\frac{1-d}{2} \delta_{i}^{k}= \\
& =\frac{1}{2} \partial_{i} E^{k}+\frac{1}{2}\left(g^{k s} \partial_{i}\left(g_{j s} E^{j}\right)-g^{k s} g_{j s} \partial_{i} E^{j}-g^{k s} \partial_{s} \theta_{i}\right)+\frac{1-d}{2} \delta_{i}^{k}= \\
& =\frac{1}{2}\left(g^{k s} \partial_{i} \theta_{s}-g^{k s} \partial_{s} \theta_{i}\right)+\frac{1-d}{2} \delta_{i}^{k}= \\
& =\frac{1}{2} g^{k s} d \theta_{i s}+\frac{1-d}{2} \delta_{i}^{k} .
\end{aligned}
$$

In the Egorov case $d \theta=0$ this formula reduces to Dubrovin's formula.

5.2. The semisimple case. Let us analyze what Theorem 5.4 gives us in the case of a semisimple flat pencil of metrics. Semisimplicity means that there are special coordinates $u^{1}, \ldots, u^{n}$ such that

$$
\eta^{i j}=f^{i} \delta_{j}^{i}, \quad g^{i j}=f^{i} u^{i} \delta_{j}^{i} .
$$

It is easy to check that the pencil is semisimple if and only if the eigenvalues of $L$ are functionally independent, and then these eigenvalues can be taken as coordinates $u^{1}, \ldots, u^{n}$. Using exactness 
it is easy to check that in the coordinates $u^{1}, \ldots, u^{n}$ the unit vector field and the Euler vector field read

$$
e^{i}=1, \quad E^{i}=u^{i}, \quad i=1, \ldots, n .
$$

Equations (5.3) and (5.4) imply that $\Delta_{i}^{j k}=0$ for $i \neq j$, and then we compute

$$
\Delta_{j}^{j k}=\left\{\begin{array}{ll}
\frac{u^{j}-u^{k}}{2^{2}} \frac{f^{k} \partial_{k} f^{j}}{f^{j}}, & \text { if } k \neq j, \\
\frac{f^{j}}{2}, & \text { if } j=k,
\end{array} \quad R_{j}^{k}=\frac{1}{f^{j}} \Delta_{j}^{j k}= \begin{cases}\frac{u^{j}-u^{k}}{2} \frac{f^{k} \partial_{k} f^{j}}{\left(f^{j}\right)^{2}}, & \text { if } k \neq j, \\
\frac{1}{2}, & \text { if } j=k .\end{cases}\right.
$$

From (5.5), under the assumption $\operatorname{det} R \neq 0$, we immediately get that $c_{j k}^{i}=\delta_{j}^{i} \delta_{k}^{i}$.

Actually, in the semisimple case we don't need the assumption $\operatorname{det} R \neq 0$ : we can directly define

$$
c_{j k}^{i}:=\delta_{j}^{i} \delta_{k}^{i}
$$

This product is clearly compatible with the metric $\eta$ and satisfies the condition $\mathcal{L}_{E} \mathrm{O}=0$. Thus, the data $(\circ, \eta, e, E)$ defines a homogeneous flat Riemannian F-manifold with Killing unit vector field.

We conclude that an arbitrary semisimple exact homogeneous flat pencil of metrics gives a homogeneous flat Riemannian F-manifold with Killing unit vector field. The converse statement is clearly not true since the existence of a second compatible flat metric requires the fulfillment of the additional constraints Mok17

$$
u^{i} \partial_{i} \beta_{j i}+u^{j} \partial_{j} \beta_{i j}+\sum_{k \neq i, j} u^{k} \beta_{i k} \beta_{j k}=-\frac{1}{2}\left(\beta_{i j}+\beta_{j i}\right), \quad i \neq j .
$$

Using the previous conditions these constraints can be replaced by the following set of $\frac{n(n-1)}{2}$ constraints:

$$
\left.\sum_{k \neq i, j}\left[u^{i}\left(u^{j}-u^{k}\right)(\Delta \beta)_{i k} \beta_{j k}-u^{j}\left(u^{i}-u^{k}\right)(\Delta \beta)_{j k} \beta_{i k}\right)\right]=\frac{1}{2}\left(u^{i}+u^{j}\right)(\Delta \beta)_{i j}, \quad i \neq j .
$$

Summarizing, semisimple exact homogeneuos flat pencils are obtained by solutions of the system (4.14.2 4.3) subject to the $n(n-1)$ constraints (4.8) and (5.6).

5.3. Semisimple exact homogeneous flat pencils in dimension 3. Let us consider the three-dimensional case. We need to study the same system studied in Section 4.1 for the functions $F_{i j}$ subject to the additional constraints (5.6), which, in terms of the functions $F_{i j}$, can be written as

$$
\left[\begin{array}{l}
Q_{6} \\
Q_{7} \\
Q_{8}
\end{array}\right]=\left[\begin{array}{ccc}
-\frac{1}{2} \frac{u^{1}+u^{2}}{u^{2}-u^{1}} & -\frac{u^{1} F_{23}}{u^{3}-u^{1}} & \frac{u^{2} F_{13}}{u^{3}-u^{2}} \\
-\frac{u^{2} F_{31}}{u^{2}-u^{1}} & \frac{u^{3} F_{21}}{u^{3}-u^{1}} & -\frac{1}{2} \frac{u^{2}+u^{3}}{u^{3}-u^{2}} \\
-\frac{u^{1} F_{32}}{u^{2}-u^{1}} & \frac{1}{2} \frac{u^{3}+u^{1}}{u^{3}-u^{1}} & \frac{u^{3} F_{12}}{u^{3}-u^{2}}
\end{array}\right]\left[\begin{array}{l}
(\Delta F)_{12} \\
(\Delta F)_{13} \\
(\Delta F)_{23}
\end{array}\right]=\left[\begin{array}{l}
0 \\
0 \\
0
\end{array}\right]
$$

These can be replaced by the constraints

$$
\begin{aligned}
& I_{6}=Q_{6}-u^{1} I_{3}=0 \\
& I_{7}=Q_{7}-u^{2} I_{4}=0 \\
& I_{8}=Q_{8}-u^{3} I_{5}=0
\end{aligned}
$$

that is

$$
\left[\begin{array}{l}
I_{6} \\
I_{7} \\
I_{8}
\end{array}\right]=\left[\begin{array}{ccc}
-\frac{1}{2} & 0 & \frac{F_{13}}{z-1} \\
0 & \frac{F_{21}(z-1)}{z} & -\frac{1}{2} \\
F_{32} z & -\frac{1}{2} & 0
\end{array}\right]\left[\begin{array}{l}
(\Delta F)_{12} \\
(\Delta F)_{13} \\
(\Delta F)_{23}
\end{array}\right]=\left[\begin{array}{l}
0 \\
0 \\
0
\end{array}\right]
$$


where we recall that $z=\frac{u^{3}-u^{1}}{u^{2}-u^{1}}$. Assuming $\beta_{i j} \neq \beta_{j i}$ for some $i \neq j$, the solution of the algebraic system $I_{3}=I_{4}=I_{5}=I_{6}=I_{7}=I_{8}=0$ is given by

$$
\begin{aligned}
& F_{21}=\frac{1}{2} \frac{\sqrt{z-1}}{\sqrt{-z}}, \\
& F_{31}=\frac{1}{2} \sqrt{z-1}, \\
& F_{12}=\frac{1}{2} \frac{\sqrt{-z}}{\sqrt{z-1}}, \\
& F_{32}=\frac{1}{2} \sqrt{-z}, \\
& F_{13}=-\frac{1}{2} \sqrt{z-1}, \\
& F_{23}=-\frac{1}{2} \frac{1}{\sqrt{-z}} .
\end{aligned}
$$

It is easy to check that the above functions satisfy the system of ODEs for the functions $F_{i j}$ and that the value of the first integrals $I_{1}$ and $I_{2}$ on this solution is $\frac{3}{4}$ and $\frac{1}{4}$, respectively. The eigenvalues of the matrix $V$ are 1 and $-\frac{1}{2}$ (with multiplicity 2). This means that if $d=1,-\frac{1}{2}$ the overdetermined system for the Lamé coefficients (4.4,4.5,4.6) admits solutions.

For $d=1$ we get

$$
H_{1}=c \sqrt{u^{3}-u^{1}} \sqrt{u^{2}-u^{1}}, \quad H_{2}=-c \sqrt{u^{1}-u^{2}} \sqrt{u^{3}-u^{2}}, \quad H_{3}=c \sqrt{u^{2}-u^{3}} \sqrt{u^{1}-u^{3}} .
$$

This example can be immediately generalized to arbitrary dimensions leading to the pair of flat diagonal metrics

$$
\left(g_{1}\right)_{i i}=\prod_{k \neq i}\left(u^{k}-u^{i}\right), \quad\left(g_{2}\right)_{i i}=\frac{1}{u^{i}} \prod_{k \neq i}\left(u^{k}-u^{i}\right) .
$$

The corresponding bi-flat F-manifold structure is the special case of Lauricella bi-flat F-manifolds corresponding to the choice $\epsilon_{i}=\frac{1}{2}, i=1, \ldots, n$. The metrics of this example provide two local Hamiltonian structures of hydrodynamic type for the quasi-classical limit of coupled $\mathrm{KdV}$ equations AF87, FP91].

For $d=-\frac{1}{2}$ we get

$$
\begin{aligned}
& H_{1}=\frac{c_{1} P_{-\frac{1}{2}}^{1}\left(\frac{2 u^{1}-u^{3}-u^{2}}{u^{2}-u^{3}}\right)+c_{2} Q_{-\frac{1}{2}}^{1}\left(\frac{2 u^{1}-u^{3}-u^{2}}{u^{2}-u^{3}}\right)}{\sqrt{u^{2}-u^{3}}}, \\
& H_{2}=-\frac{c_{1}}{2} \frac{P_{-\frac{1}{2}}^{1}\left(\frac{2 u^{1}-u^{3}-u^{2}}{u^{2}-u^{3}}\right)+P_{\frac{1}{2}}^{1}\left(\frac{2 u^{1}-u^{3}-u^{2}}{u^{2}-u^{3}}\right)}{\sqrt{u^{3}-u^{1}}}-\frac{c_{2}}{2} \frac{Q_{-\frac{1}{2}}^{1}\left(\frac{2 u^{1}-u^{3}-u^{2}}{u^{2}-u^{3}}\right)+Q_{\frac{1}{2}}^{1}\left(\frac{2 u^{1}-u^{3}-u^{2}}{u^{2}-u^{3}}\right)}{\sqrt{u^{3}-u^{1}}}, \\
& H_{3}=\frac{c_{1}}{2} \frac{P_{-\frac{1}{2}}^{1}\left(\frac{2 u^{1}-u^{3}-u^{2}}{u^{2}-u^{3}}\right)-P_{\frac{1}{2}}^{1}\left(\frac{2 u^{1}-u^{3}-u^{2}}{u^{2}-u^{3}}\right)}{\sqrt{u^{3}-u^{1}}}+\frac{c_{2}}{2} \frac{Q_{-\frac{1}{2}}^{1}\left(\frac{2 u^{1}-u^{3}-u^{2}}{u^{2}-u^{3}}\right)-Q_{\frac{1}{2}}^{1}\left(\frac{2 u^{1}-u^{3}-u^{2}}{u^{2}-u^{3}}\right)}{\sqrt{u^{1}-u^{2}}},
\end{aligned}
$$

where $P_{\nu}^{\mu}(x)$ and $Q_{\nu}^{\mu}(x)$ are Legendre functions of the first and second kind respectively, i.e. are solutions of the general Legendre equation

$$
\left(1-x^{2}\right) y^{\prime \prime}-2 x y^{\prime}+\left[\nu(\nu+1)-\frac{\mu^{2}}{1-x^{2}}\right] y=0 .
$$

\section{LEGENDRE TRANSFORMATIONS}

Throughout this section we will not assume that F-manifolds have unit.

\subsection{The Legendre transformation for F-manifolds with compatible connection.}

Definition 6.1. An F-manifold with compatible connection [LPR11] is a manifold $M$ equipped with an associative commutative product $\circ$ and a connection $\nabla$ satisfying the following conditions:

- $\nabla$ is torsionless and compatible with the product $\circ$.

- The Riemann tensor $R$ of $\nabla$ satisfies the condition

$$
R(Y, Z)(X \circ W)+R(X, Y)(Z \circ W)+R(Z, X)(Y \circ W)=0 .
$$


Given a semisimple F-manifold with compatible connection one can define an integrable hierarchy

$$
u_{t}^{i}=c_{j k}^{i} X^{j} u_{x}^{k}, \quad i=1, \ldots, n,
$$

where the $X^{j}$ are components of a vector field $X$ satisfying the linear system of PDEs

$$
c_{j l}^{i} \nabla_{k} X^{l}=c_{k l}^{i} \nabla_{j} X^{l}
$$

Non-trivial solutions of this system exist because of semisimplicity [LPR11, Section 5]. If the connection $\nabla$ is flat, then condition (6.1) is automatically satisfied. In this case a countable set of solutions of (6.3) is obtained starting from a frame of flat vector fields $X_{(p, 0)}, p=1, \ldots, n$, by means of the following recursive relations:

$$
\nabla_{j} X_{(p, \alpha+1)}^{i}=c_{j k}^{i} X_{(p, \alpha)}^{k} .
$$

This was called the principal hierarchy, since in the case of Dubrovin-Frobenius manifolds it reduces to Dubrovin's principal hierarchy [LPR11.

Recall that an invertible vector field $X$ is a vector field for which there exists another vector field $Y$ such that $X \circ Y=e$. Following [Ste17, SS17], for any invertible vector field $\bar{X}$ solving the linear system (6.3) we can define a generalized Legendre transformation. We will call such a vector field a Legendre vector field.

Theorem 6.2. Let $(M, \circ, \nabla)$ be an F-manifold with compatible connection and let $\bar{X}$ be an invertible vector field satisfying condition (6.3). Then the data $(M, \circ, \bar{\nabla})$, where $\bar{\nabla}$ is the connection defined by

$$
\bar{\nabla}_{Y} Z:=\bar{X}^{-1} \circ \nabla_{Y}(\bar{X} \circ Z)
$$

give an F-manifold with compatible connection.

Proof. The statement of the theorem follows from the following facts proved in [Ste17, SS17]:

- The connection $\bar{\nabla}$ is compatible with the product $\circ$ if and only if $\nabla$ is compatible with the product $\circ$ and $\bar{X}$ satisfies condition (6.3).

- The torsion of $\bar{\nabla}$ vanishes as a consequence of the vanishing of the torsion of $\nabla$ and of condition (6.3).

- If $\bar{X}$ satisfies condition (6.3), then the Riemann tensor $R$ of $\nabla$ and the Riemann tensor $\bar{R}$ of $\bar{\nabla}$ are related by the following identity:

$$
\bar{R}(Y, Z)(W)=\bar{X}^{-1} \circ R(Y, Z)(\bar{X} \circ W) .
$$

Remark 6.3. If $\nabla$ is flat then $\bar{\nabla}$ is flat too. Moreover, if the product $\circ$ has a unit $e$ and $\nabla \bar{X}=0$, then $\bar{\nabla} e=0$.

Remark 6.4. In canonical coordinates the Legendre transformation is given by the following formulas:

$$
\begin{array}{ll}
\bar{\Gamma}_{i j}^{i}=\Gamma_{i j}^{i} \frac{\bar{X}^{j}}{\bar{X}^{i}}=\Gamma_{i j}^{i}+\partial_{j} \ln \bar{X}^{i}, & i \neq j, \\
\bar{\Gamma}_{j j}^{i}=\Gamma_{j j}^{i} \frac{\bar{X}^{j}}{\bar{X}^{i}}=-\Gamma_{i j}^{i}-\partial_{j} \ln \bar{X}^{i}, & i \neq j, \\
\bar{\Gamma}_{i i}^{i}=\Gamma_{i i}^{i}+\partial_{i} \ln \bar{X}^{i}, & \\
\bar{\Gamma}_{j k}^{i}=0, & i \neq j \neq k \neq i,
\end{array}
$$

where we have used the fact that

$$
\partial_{j} \bar{X}^{i}=\Gamma_{i j}^{i}\left(\bar{X}^{j}-\bar{X}^{i}\right), \quad i \neq j .
$$




\subsection{The Legendre transformation for Riemannian F-manifolds.}

Theorem 6.5. Let $(M, \circ, g, e)$ be a (pseudo-)Riemannian F-manifold with Killing unit vector field and $\nabla$ be the associated flat structure on $M$. If a Legendre vector field $\bar{X}$ is flat, i.e. $\nabla \bar{X}=0$, then the data $(M, \circ, e, \bar{g})$, where $\bar{g}$ is given by

$$
\bar{g}(Y, Z):=g(\bar{X} \circ Y, \bar{X} \circ Z), \quad Y, Z \in \mathcal{T}_{M},
$$

define a new (pseudo-)Riemannian F-manifold structure with Killing unit vector field on $M$ whose associated flat structure is $\bar{\nabla}$.

Proof. To prove invariance of the metric $\bar{g}$, we compute

$$
\bar{g}(Y \circ Z, W)=g(\bar{X} \circ Y \circ Z, \bar{X} \circ W)=g(\bar{X} \circ Y, \bar{X} \circ W \circ Z)=\bar{g}(Y, W \circ Z),
$$

as required.

To prove that $\bar{\nabla}$ is the flat connection associated to $\bar{g}$ in the sense of Theorem 1.8, we have to prove that

$$
\left(\bar{\nabla}_{Y} \bar{g}\right)(W, Z)=\frac{1}{2} d \bar{\theta}(Y \circ W, Z)+\frac{1}{2} d \bar{\theta}(Y \circ Z, W)
$$

Note that

$$
d \bar{\theta}(W, Z)=W(\bar{\theta}(Z))-Z(\bar{\theta}(W))-\bar{\theta}([W, Z])=\left(\bar{\nabla}_{W} \bar{g}\right)(e, Z)-\left(\bar{\nabla}_{Z} \bar{g}\right)(e, W) .
$$

Therefore, condition (6.5) is equivalent to

$$
\left(\bar{\nabla}_{Y} \bar{g}\right)(W, Z)=\frac{1}{2}\left(\bar{\nabla}_{Y \circ W} \bar{g}\right)(e, Z)-\frac{1}{2}\left(\bar{\nabla}_{Z} \bar{g}\right)(e, Y \circ W)+\frac{1}{2}\left(\bar{\nabla}_{Y \circ Z} \bar{g}\right)(e, W)-\frac{1}{2}\left(\bar{\nabla}_{W} \bar{g}\right)(e, Y \circ Z) .
$$

To prove this we compute

$$
\begin{aligned}
\left(\bar{\nabla}_{Y} \bar{g}\right)(W, Z)= & Y(g(\bar{X} \circ W, \bar{X} \circ Z))-\bar{g}\left(\bar{\nabla}_{Y} W, Z\right)-\bar{g}\left(W, \bar{\nabla}_{Y} Z\right)= \\
= & \left(\nabla_{Y} g\right)(\bar{X} \circ W, \bar{X} \circ Z)+g\left(\left(\nabla_{Y} c\right)(\bar{X}, W), \bar{X} \circ Z\right)+g\left(\bar{X} \circ \nabla_{Y} W, \bar{X} \circ Z\right) \\
& +g\left(\bar{X} \circ W,\left(\nabla_{Y} c\right)(\bar{X}, Z)\right)+g\left(\bar{X} \circ W, \bar{X} \circ \nabla_{Y} Z\right)-\bar{g}\left(\bar{\nabla}_{Y} W, Z\right)-\bar{g}\left(W, \bar{\nabla}_{Y} Z\right)= \\
= & \left(\nabla_{Y} g\right)(\bar{X} \circ W, \bar{X} \circ Z)+g\left(\left(\nabla_{Y} c\right)(\bar{X}, W), \bar{X} \circ Z\right)+g\left(\bar{X} \circ \nabla_{Y} W, \bar{X} \circ Z\right) \\
& +g\left(\bar{X} \circ W,\left(\nabla_{Y} c\right)(\bar{X}, Z)\right)+g\left(\bar{X} \circ W, \bar{X} \circ \nabla_{Y} Z\right)-\bar{g}\left(\nabla_{Y} W, Z\right)-\bar{g}\left(W, \nabla_{Y} Z\right) \\
& -\bar{g}\left(\bar{X}^{-1} \circ\left(\nabla_{Y} c\right)(\bar{X}, W), Z\right)-\bar{g}\left(W, \bar{X}^{-1} \circ\left(\nabla_{Y} c\right)(\bar{X}, Z)\right)= \\
= & \left(\nabla_{Y} g\right)(\bar{X} \circ W, \bar{X} \circ Z) .
\end{aligned}
$$

Therefore, equation (6.6) is equivalent to

$$
\begin{aligned}
\left(\nabla_{Y} g\right)(\bar{X} \circ W, \bar{X} \circ Z)= & \frac{1}{2}\left(\nabla_{Y \circ W} g\right)(\bar{X}, \bar{X} \circ Z)-\frac{1}{2}\left(\nabla_{Z} g\right)(\bar{X}, \bar{X} \circ Y \circ W) \\
& +\frac{1}{2}\left(\nabla_{Y \circ Z} g\right)(\bar{X}, \bar{X} \circ W)-\frac{1}{2}\left(\nabla_{W} g\right)(\bar{X}, \bar{X} \circ Y \circ Z) .
\end{aligned}
$$

Using that $\left(\nabla_{Y} g\right)(W, Z)=\frac{1}{2} d \theta(Y \circ W, Z)+\frac{1}{2} d \theta(Y \circ Z, W)$, for the left-hand side of (6.7) we obtain

$$
\frac{1}{2} d \theta(Y \circ \bar{X} \circ W, \bar{X} \circ Z)+\frac{1}{2} d \theta(Y \circ \bar{X} \circ Z, \bar{X} \circ W)
$$


while for the right-hand side of (6.7) we get

$$
\begin{aligned}
& \frac{1}{4} d \theta(Y \circ W \circ \bar{X}, \bar{X} \circ Z)+\frac{1}{4} d \theta(Y \circ W \circ \bar{X} \circ Z, \bar{X})-\frac{1}{4} d \theta(Z \circ \bar{X}, \bar{X} \circ Y \circ W) \\
& -\frac{1}{4} d \theta(Z \circ \bar{X} \circ Y \circ W, \bar{X})+\frac{1}{4} d \theta(Y \circ Z \circ \bar{X}, \bar{X} \circ W)+\frac{1}{4} d \theta(Y \circ Z \circ \bar{X} \circ W, \bar{X}) \\
& -\frac{1}{4} d \theta(W \circ \bar{X}, \bar{X} \circ Y \circ Z)-\frac{1}{4} d \theta(W \circ \bar{X} \circ Y \circ Z, \bar{X})= \\
= & \frac{1}{2} d \theta(Y \circ \bar{X} \circ W, \bar{X} \circ Z)+\frac{1}{2} d \theta(Y \circ \bar{X} \circ Z, \bar{X} \circ W),
\end{aligned}
$$

as required.

The fact that the Riemann tensor of $\bar{g}$ satisfies condition (1.4) follows now from the fact that $\bar{\nabla}$ is flat and Proposition 1.12 .

The property $\mathcal{L}_{e} \bar{g}=0$ follows from Lemma 1.14.

Remark 6.6. In the semisimple case, in canonical coordinates the Lamé coefficients $\bar{H}_{i}$ of the metric $\bar{g}$ are related to the Lamé coefficients $H_{i}$ of the metric $g$ by

$$
\bar{H}_{i}=H_{i} \bar{X}^{i}, \quad i=1, \ldots, n,
$$

where $\bar{X}$ satisfies the condition

$$
\partial_{j} \bar{X}^{i}=\Gamma_{i j}^{i}\left(\bar{X}^{j}-\bar{X}^{i}\right)=\beta_{j i} \frac{H_{j}}{H_{i}}\left(\bar{X}^{j}-\bar{X}^{i}\right), \quad i \neq j .
$$

Using this fact we get $\beta_{i j}=\bar{\beta}_{i j}$. In classical differential geometry two diagonal metrics with the same rotation coefficients are said to be Combescure equivalent.

If the Legendre vector field is homogeneous, then the associated transformation preserves the homogeneity property.

Theorem 6.7. Let $(M, \circ, g, e, E)$ be a homogeneous Riemannian F-manifold with Killing unit vector field. If the Legendre vector field $\bar{X}$ is flat and homogeneous, i.e. $\mathcal{L}_{E} \bar{X}=\bar{d} \cdot \bar{X}$ for some constant $\bar{d}$, then the data $(M, \circ, \bar{g}, e, E)$ define a new homogeneous Riemannian F-manifold with Killing unit vector field.

Proof. Due to the previous theorem we only need to check homogeneity of the new metric $\bar{g}$, but this follows immediately from the hypothesis and we have $\mathcal{L}_{E} \bar{g}=(D+2 \bar{d}+2) \bar{g}$.

Below we provide an interpretation of the results from Sections 4.1 .1 and 5.3 in terms of Legendre transformations.

6.2.1. The flat F-manifolds from Section 4.1.1. Let us consider the flat structure $(\nabla, \circ, e)$ associated with the first metric obtained in Section 4.1.1. In canonical coordinates it is given by the Christoffel symbols

$$
\begin{array}{ll}
\Gamma_{12}^{1}=\frac{b}{\left(a u^{1}-a u^{3}+b u^{1}-b u^{2}\right)}, & \Gamma_{12}^{2}=-\frac{(a+b)}{\left(a u^{1}-a u^{3}+b u^{1}-b u^{2}\right)}, \\
\Gamma_{13}^{1}=\frac{a}{\left(a u^{1}-a u^{3}+b u^{1}-b u^{2}\right)}, & \Gamma_{13}^{3}=-\frac{(a+b)}{\left(a u^{1}-a u^{3}+b u^{1}-b u^{2}\right)}, \\
\Gamma_{23}^{2}=\frac{a}{\left(a u^{1}-a u^{3}+b u^{1}-b u^{2}\right)}, & \Gamma_{23}^{3}=\frac{b}{\left(a u^{1}-a u^{3}+b u^{1}-b u^{2}\right)},
\end{array}
$$

using the conditions

$$
\Gamma_{j j}^{i}=-\Gamma_{i j}^{i}=-\Gamma_{j i}^{i} \quad(i \neq j), \quad \Gamma_{j k}^{i}=0 \quad(i \neq j \neq k \neq i), \quad \Gamma_{i i}^{i}=-\sum_{j \neq i} \Gamma_{i j}^{i} .
$$


It is straightforward to check that vector fields that are flat with respect to $\nabla$ are linear combinations of the unit vector field $\bar{X}_{(1)}:=e$, of the vector field $\bar{X}_{(2)}$ with components

$$
\bar{X}_{(2)}^{1}:=u^{2}-u^{3}, \quad \bar{X}_{(2)}^{2}:=\frac{(a+b)}{b}\left(u^{1}-u^{3}\right), \quad \bar{X}_{(2)}^{3}:=\frac{(a+b)}{a}\left(u^{2}-u^{1}\right),
$$

and of the vector field $\bar{X}_{(3)}$ with components

$$
\begin{aligned}
& \bar{X}_{(3)}^{1}:=-(a+b)\left(u^{1}\right)^{2}-2\left(a u^{3}+b u^{2}\right) u^{1}-a\left(u^{3}\right)^{2}-b\left(u^{2}\right)^{2}, \\
& \bar{X}_{(3)}^{2}:=(a+b)\left(u^{1}\right)^{2}-2(a+b) u^{1} u^{2}+a u^{3}\left(2 u^{2}-u^{3}\right)+b\left(u^{2}\right)^{2}, \\
& \bar{X}_{(3)}^{3}:=(a+b)\left(u^{1}\right)^{2}-2 u^{3}(a+b) u^{1}+a\left(u^{3}\right)^{2}-b u^{2}\left(u^{2}-2 u^{3}\right) .
\end{aligned}
$$

Applying the Legendre transformations generated by the vector fields $\bar{X}_{(2)}$ and $\bar{X}_{(3)}$ to the first metric we get the second and the third metric of Section 4.1.1.

6.2.2. The F-manifolds from Section 5.3. Let us consider the flat structure $(\nabla, \circ, e)$ associated with the first metric of Section 5.3. In canonical coordinates it is given by the Christoffel symbols

$$
\Gamma_{i j}^{i}=\frac{1}{2\left(u^{j}-u^{i}\right)}, \quad i \neq j,
$$

using conditions (6.8). It is straightforward to check that vector fields that are flat with respect to $\nabla$ are linear combinations of the unit vector field $\bar{X}_{(1)}:=e$, of the vector field $\bar{X}_{(2)}$ with components

$$
\begin{aligned}
& \bar{X}_{(2)}^{1}:=\frac{P_{-\frac{1}{2}}^{1}\left(\frac{2 u^{1}-u^{3}-u^{2}}{u^{2}-u^{3}}\right)}{\sqrt{\left(u^{1}-u^{2}\right)\left(u^{1}-u^{3}\right)\left(u^{2}-u^{3}\right)}}, \\
& \bar{X}_{(2)}^{2}:=-\frac{1}{2} \frac{P_{-\frac{1}{2}}^{1}\left(\frac{2 u^{1}-u^{3}-u^{2}}{u^{2}-u^{3}}\right)+P_{\frac{1}{2}}^{1}\left(\frac{2 u^{1}-u^{3}-u^{2}}{u^{2}-u^{3}}\right)}{\sqrt{\left(u^{1}-u^{2}\right)\left(u^{1}-u^{3}\right)\left(u^{2}-u^{3}\right)}}, \\
& \bar{X}_{(2)}^{3}:=-\frac{1}{2} \frac{P_{-\frac{1}{2}}^{1}\left(\frac{2 u^{1}-u^{3}-u^{2}}{u^{2}-u^{3}}\right)-P_{\frac{1}{2}}^{1}\left(\frac{2 u^{1}-u^{3}-u^{2}}{u^{2}-u^{3}}\right)}{\sqrt{\left(u^{1}-u^{2}\right)\left(u^{1}-u^{3}\right)\left(u^{2}-u^{3}\right)}}
\end{aligned}
$$

and of the vector field $\bar{X}_{(3)}$ with components

$$
\begin{aligned}
& \bar{X}_{(3)}^{1}:=\frac{Q_{-\frac{1}{2}}^{1}\left(\frac{2 u^{1}-u^{3}-u^{2}}{u^{2}-u^{3}}\right)}{\sqrt{\left(u^{1}-u^{2}\right)\left(u^{1}-u^{3}\right)\left(u^{2}-u^{3}\right)}}, \\
& \bar{X}_{(3)}^{2}:=-\frac{1}{2} \frac{Q_{-\frac{1}{2}}^{1}\left(\frac{2 u^{1}-u^{3}-u^{2}}{u^{2}-u^{3}}\right)+Q_{\frac{1}{2}}^{1}\left(\frac{2 u^{1}-u^{3}-u^{2}}{u^{2}-u^{3}}\right)}{\sqrt{\left(u^{1}-u^{2}\right)\left(u^{1}-u^{3}\right)\left(u^{2}-u^{3}\right)}}, \\
& \bar{X}_{(3)}^{3}:=-\frac{1}{2} \frac{Q_{-\frac{1}{2}}^{1}\left(\frac{2 u^{1}-u^{3}-u^{2}}{u^{2}-u^{3}}\right)-Q_{\frac{1}{2}}^{1}\left(\frac{2 u^{1}-u^{3}-u^{2}}{u^{2}-u^{3}}\right)}{\sqrt{\left(u^{1}-u^{2}\right)\left(u^{1}-u^{3}\right)\left(u^{2}-u^{3}\right)}} .
\end{aligned}
$$

Applying the Legendre transformations generated by the linear combinations of the vector fields $\bar{X}_{(2)}$ and $\bar{X}_{(3)}$ to the first metric we get the metrics corresponding to the eigenvalue $-\frac{1}{2}$.

Remark 6.8. Some computations suggest that, even in the case $n>3$, non-Egorov homogeneous exact flat pencils of metrics are related to the flat pencil (5.7) by a Legendre transformation. 


\section{Appendix}

In dimension 2 regular non semisimple homogeneous flat structures are given by the twoparameter family considered in the Example 1.4. In this Appendix we list the associated vector potentials. We have 5 different cases depending on the values of the parameter $a$.

Case I: $a \neq-2,-1,0,1$. The flat coordinates are given by

$$
u=x-\frac{b}{a} y, \quad v=\frac{y^{a+1}}{a+1} .
$$

In these coordinates we have

$$
e=\partial_{u}, \quad E=u \partial_{u}+(a+1) v \partial_{v}
$$

and the vector potential reads

$$
F^{1}=\frac{a^{2}(a-1) u^{2}+b^{2}(a+1)^{\frac{2}{a+1}} v^{\frac{2}{a+1}}}{2(a-1) a^{2}}, \quad F^{2}=\frac{a(a+2) u v+2 b(a+1)^{\frac{a+2}{a+1}} v^{\frac{a+2}{a+1}}}{(a+2) a} .
$$

Case II: $a=-2$. The flat coordinates are given by

$$
u=x+\frac{1}{2} b y, \quad v=-\frac{1}{y} .
$$

In these coordinates we have

$$
e=\partial_{u}, \quad E=u \partial_{u}-v \partial_{v}
$$

and the vector potential reads

$$
F^{1}=\frac{1}{2} u^{2}-\frac{1}{24} \frac{b^{2}}{v^{2}}, \quad F^{2}=u v+b \ln v .
$$

Case III: $a=-1$. The flat coordinates are given by

$$
u=x+b y, \quad v=\ln y .
$$

In these coordinates we have

$$
e=\partial_{u}, \quad E=u \partial_{u}+\partial_{v}
$$

and the vector potential reads

$$
F^{1}=\frac{1}{2} u^{2}-\frac{1}{4} b^{2} e^{2 v}, \quad F^{2}=u v-2 b e^{v} .
$$

Case IV: $a=0$. The flat coordinates are given by

$$
u=x+b y \ln y, \quad v=y .
$$

In these coordinates we have

$$
e=\partial_{u}, \quad E=(u+b v) \partial_{u}+v \partial_{v}
$$

and the vector potential reads

$$
F^{1}=\frac{1}{2} u^{2}-\frac{1}{2} b^{2} v^{2}(\ln v)^{2}+\frac{1}{2} b^{2} v^{2} \ln v-\frac{3}{4} b^{2} v^{2}, \quad F^{2}=-b v^{2} \ln v+\frac{1}{2} b v^{2}+u v .
$$

Case V: $a=1$. The flat coordinates are given by

$$
u=x-b y, \quad v=\frac{y^{2}}{2} .
$$


In these coordinates we have

$$
e=\partial_{u}, \quad E=u \partial_{u}+2 v \partial_{v}
$$

and the vector potential reads

$$
F^{1}=\frac{1}{2} u^{2}-\frac{1}{2} b^{2} v \ln v+\frac{1}{2} b^{2} v, \quad F^{2}=\frac{4}{3} b \sqrt{2} v^{\frac{3}{2}}+u v .
$$

\section{REFERENCES}

[AF87] M. Antonowicz, A. P. Fordy. Coupled KdV equations with multi-Hamiltonian structures. Physica D 28 (1987), no. 3, 345-357.

[ABLR20a] A. Arsie, A. Buryak, P. Lorenzoni, P. Rossi. Semisimple flat F-manifolds in higher genus. arXiv:2001.05599.

[ABLR20b] A. Arsie, A. Buryak, P. Lorenzoni, P. Rossi. Flat F-manifolds, F-CohFTs, and integrable hierarchies. arXiv:2012.05332.

[AL13] A. Arsie, P. Lorenzoni. From the Darboux-Egorov system to bi-flat F-manifolds. Journal of Geometry and Physics 70, (2013), 98-116.

[AL17] A. Arsie, P. Lorenzoni. Complex reflection groups, logarithmic connections and bi-flat F-manifolds. Letters in Mathematical Physics 107 (2017), no. 10, 1919-1961.

[AL18] A. Arsie, P. Lorenzoni. Flat F-manifolds, Miura invariants, and integrable systems of conservation laws. Journal of Integrable Systems 3 (2018), no. 1, xyy004.

[AL19] A. Arsie, P. Lorenzoni. F-manifolds, multi-flat structures and Painlevé transcendents. Asian Journal of Mathematics 23 (2019), no. 5, 877-904.

[BB19] A. Basalaev, A. Buryak. Open WDVV equations and Virasoro constraints. Arnold Mathematical Journal 5 (2019), no. 2-3, 145-186.

[Bur15] A. Buryak. Double ramification cycles and integrable hierarchies. Communications in Mathematical Physics 336 (2015), no. 3, 1085-1107.

[BR16] A. Buryak, P. Rossi. Recursion relations for double ramification hierarchies. Communications in Mathematical Physics 342 (2016), no. 2, 533-568.

[BR18] A. Buryak, P. Rossi. Extended r-spin theory in all genera and the discrete KdV hierarchy. arXiv:1806.09825

[CLR10] G. Carlet, P. Lorenzoni, A. Raimondo. The reductions of the dispersionless $2 D$ Toda hierarchy and their Hamiltonian structures. Journal of Physics. A. Mathematical and Theoretical 43 (2010), no. 4, 045201 .

[CHL05] W. Couwenberg, G. Heckman, E. Looijenga. Geometric structures on the complement of a projective arrangement. Publications Mathématiques. Institut de Hautes Études Scientifiques 101 (2005), 69161.

[Dar10] G. Darboux. Leçons sur les systémes orthogonaux et les coordonnées curvilignes. Gauthier-Villars, Paris, 1910.

[DH17] L. David, C. Hertling. Regular F-manifolds: initial conditions and Frobenius metrics. Annali della Scuola Normale Superiore di Pisa. Classe di Scienze. Serie V 17 (2017), no. 3, 1121-1152.

[DH19] L. David, C. Hertling. Meromorphic connections over F-manifolds. arXiv:1912.03331.

[DS11] L. David, I. A. B. Strachan. Dubrovin's duality for F-manifolds with eventual identities. Advances in Mathematics 226 (2011), no. 5, 4031-4060.

[Dub90] B. A. Dubrovin. Differential geometry of strongly integrable systems of hydrodynamic type. Functional Analysis and Its Applications 24 (1990), 280-285.

[Dub96] B. Dubrovin. Geometry of $2 D$ topological field theories. In: Integrable systems and quantum groups (Montecatini Terme, 1993), 120-348, Lecture Notes in Math., 1620, Fond. CIME/CIME Found. Subser., Springer, Berlin, 1996.

[Dub98] B. Dubrovin. Flat pencils of metrics and Frobenius manifolds. In: Integrable systems and algebraic geometry (Kobe/Kyoto, 1997), 47-72, World Sci. Publ., River Edge, NJ, 1998.

[Dub98] B. Dubrovin. Differential geometry of the space of orbits of a Coxeter group. Surveys in Differential Geometry 4 (1998), 181-211.

[DN84] B. A. Dubrovin, S. P. Novikov. On Poisson brackets of hydrodynamic type. Soviet Mathematics. Doklady 30 (1984), 651-654.

[Ego70] D. F. Egorov. Works in Differential Geometry (in Russian). Nauka, Moscow, 1970.

[Fer91] E. V. Ferapontov. Differential geometry of nonlocal Hamiltonian operators of hydrodynamic type. Functional Analysis and its Applications 25 (1991), no. 3, 195-204.

[FP91] E. V. Ferapontov, M. V. Pavlov. Quasiclassical limit of coupled KdV equations. Riemann invariants and multi-Hamiltonian structure. Physica D 52 (1991), no. 2-3, 211-219. 
[Get04] E. Getzler. The jet-space of a Frobenius manifold and higher-genus Gromov-Witten invariants. In: Frobenius manifolds, 45-89, Aspects Math., E36, Friedr. Vieweg, Wiesbaden, 2004.

[GLR09] J. Gibbons, P. Lorenzoni, A. Raimondo. Hamiltonian structures of reductions of the Benney system. Communications in Mathematical Physics 287 (2009), no. 1, 291-322.

[Hert02] C. Hertling. Frobenius manifolds and moduli spaces for singularities. Cambridge Tracts in Math., 151. Cambridge University Press, Cambridge, 2002.

[HM99] C. Hertling, Yu. Manin. Weak Frobenius manifolds. International Mathematics Research Notices 1999 (1999), no. 6, 277-286.

[KMS20] M. Kato, T. Mano, J. Sekiguchi. Flat structure on the space of isomonodromic deformations. Symmetry, Integrability and Geometry: Methods and Applications 16 (2020), paper no. 110.

[KM19] H. Kawakami, T. Mano. Regular flat structure and generalized Okubo system. Communications in Mathematical Physics 369 (2019), no. 2, 403-431.

[KMS18] Y. Konishi, S. Minabe, Y. Shiraishi. Almost duality for Saito structure and complex reflection groups. Journal of Integrable Systems 3 (2018), no. 1, xyy003.

[Lau1893] G. Lauricella. Sulle funzioni ipergeometriche a piú variabili. Rendiconti del Circolo Matematico di Palermo VII (1893), 111-158.

[Loo07] E. Looijenga. Uniformization by Lauricella functions - an overview of the theory of Deligne-Mostow. In: Arithmetic and geometry around hypergeometric functions, Prog. Math. 260, Birkhäuser, Basel (2007), 207-244.

[Lor14] P. Lorenzoni. Darboux-Egorov System, bi-flat F-manifolds and Painlevé VI. International Mathematics Research Notices 2014 (2014), no. 12, 3279-3302.

[LP11] P. Lorenzoni, M. Pedroni. Natural connections for semi-Hamiltonian systems: the case of the $\varepsilon$ system. Letters in Mathematical Physics 97 (2011), no. 1, 85-108.

[LPR11] P. Lorenzoni, M. Pedroni, A. Raimondo. F-manifolds and integrable systems of hydrodynamic type. Archivum Mathematicum 47 (2011), no. 3, 163-180.

[Man05] Y. I. Manin. F-manifolds with flat structure and Dubrovin's duality. Advances in Mathematics 198 (2005), no. 1, 5-26.

[Mok17] O. Mokhov. Pencils of compatible metrics and integrable systems. Russian Mathematical Surveys 72 (2017), no. 5, 889-937.

[PT03] M. V. Pavlov, S. P. Tsarev. Tri-Hamiltonian structures of Egorov systems of hydrodynamic type. Functional Analysis and Its Applications 37 (2003), no. 1, 32-45.

[Sab98] C. Sabbah. Frobenius manifolds: Isomonodromic deformations and infinitesimal period mappings. Expositiones Mathematicae 16 (1998), no. 1, 1-57.

[Sai93] K. Saito. On a linear structure of the quotient variety by a finite reflexion group. Publications of the Research Institute for Mathematical Sciences, Kyoto University 29 (1993), no. 4, 535-579.

[SYS80] K. Saito K., T. Yano, J. Sekiguchi. On a certain generator system of the ring of invariants of a finite reflection group. Communications in Algebra 8 (1980), no. 4, 373-408.

[Ser91] D. Serre. Richness and the classification of quasilinear hyperbolic systems. In: Multidimensional hyperbolic problems and computations (Minneapolis, MN, 1989), 315-333, IMA Vol. Math. Appl., 29, Springer, New York, 1991.

[Ste17] R. J. Stedman. Deformations, extensions and symmetries of solutions to the WDVV equations. Phd thesis, University of Glasgow, 2017.

[SS17] I. A. B. Strachan, R. Stedman. Generalized Legendre transformations and symmetries of the WDVV equations. Journal of Physics A: Mathematical and Theoretical 50 (2017), no. 9, 095202.

[Tsa86] S. P. Tsarev. PhD thesis. Moscow State University, 1986.

[Tsa91] S. P. Tsarev. The geometry of Hamiltonian systems of hydrodynamic type. The generalised hodograph method. Mathematics of the USSR-Izvestiya 37 (1991), no. 2, 397-419.

A. ARsie:

Department of Mathematics and Statistics, The University of Toledo, 2801W. Bancroft St., 43606 Toledo, OH, USA

Email address: alessandro.arsie@utoledo.edu

A. BURYAK:

Faculty of Mathematics, National Research University Higher School of Economics, 6 Usacheva str., Moscow, 119048, Russian Federation;

Center for Advanced Studies, Skolkovo Institute of Science and Technology, 1 Nobel str., Moscow, 143026, Russian Federation; 
Novosibirsk State University,

1 Pirogova str., Novosibirsk, 630090, Russian Federation

Email address: aburyak@hse.ru

P. LORENZONI:

Dipartimento di Matematica e Applicazioni, Università di Milano-Bicocca, Via Roberto Cozzi 53, I-20125 Milano, Italy and INFN sezione di Milano-Bicocca

Email address: paolo.lorenzoni@unimib.it

P. Rossi:

Dipartimento di Matematica "Tullio Levi-Civita", Università degli Studi di Padova, Via Trieste 63, 35121 Padova, Italy

Email address: paolo.rossi@math.unipd.it 